\title{
Entropy-induced Microphase Separation in Hard Diblock Copolymers
}

\author{
Paul P. F. Wessel.* and Bela M. Mulder \\ FOM Institute for Atomic and Molecular Physics, \\ Kruislaan 407, 1098 SJ Amsterdam, The Netherlands
}

(Dated: November 1, 2018)

\begin{abstract}
Whereas entropy can induce phase behavior that is as rich as seen in energetic systems, microphase separation remains a very rare phenomenon in entropic systems. In this paper, we present a density functional approach to study the possibility of entropy-driven microphase separation in diblock copolymers. Our model system consists of copolymers composed of freely-jointed slender hard rods. The two types of monomeric segments have comparable lengths, but a significantly different diameter, the latter difference providing the driving force for the phase separation. At the same time these systems can also exhibit liquid crystalline phases. We treat this system in the appropriate generalization of the Onsager approximation to chain-like particles. Using a linear stability (bifurcation) analysis, we analytically determine the onset of the microseparated and the nematic phases for long chains. We find that for very long chains the microseparated phase always preempts the nematic. In the limit of infinitely long chains, the correlations within the chain become Gaussian and the approach becomes exact. This allows us to define a Gaussian limit in which the theory strongly simplifies and the competition between microphase separation and liquid crystal formation can be studied essentially analytically. Our main results are phase diagrams as a function of the effective diameter difference, the segment composition and the length ratio of the segments. We also determine the amplitude of the positional order as a function of position along the chain at the onset of the microphase separation instability. Finally, we give suggestions as to how this type of entropy-induced microphase separation could be observed experimentally.
\end{abstract}

\section{INTRODUCTION}

Microphase separation (MPS) is the phenomenon where an initially homogeneous phase develops an inhomogeneous spatial structure on a microscopic scale. Usually such systems consist in part of thermodynamically incompatible components that left by themselves would tend to (macroscopically) phase separate. However, due to additional constraints of a physical or chemical nature the spatial separation between the components is prevented from increasing beyond a microscopic length scale. This leads to phases in which the components can demix only locally. There are a few archetypical examples of systems showing MPS: (i) Two (usually flexible) polymers species that have an unfavourable mutual interaction energy which are joined together by a chemical bond. This type of block copolymers [1, 2, 3] shows a wealth of microphases. (ii) Side-chain liquid crystalline polymers (LCPs). Here, liquid crystal-forming groups are linked to polymer backbones through flexible spacers. The most prominent phase of these systems is the smectic, where the LC groups form orientationally ordered layers separated by disordered lamellae containing the poymeric backbones [4, 5]. (iii) Ternary systems consisting of water, oil and an amphiphilic surfactant. These systems can show a variety of microstructured phases, with the amphiphilic surfactant stabilizing the oil-water interfaces

\footnotetext{
*Electronic address: wessels@thphy.uni-duesseldorf.nl Present address: Heinrich-Heine-Universität Düsseldorf, Institut für Theoretische Physik II Universitätsstraße 1, Gebäude 25.32, D-40225 Düsseldorf, Germany
}

and thus preventing "macrophase separation" [6, 7]. All three of the cases above are examples of thermotropic systems, i.e. systems in which the phase behavior is governed by temperature as a controlling variable, reflecting the dominance of energetic effects.

Recently, MPS was observed in an entirely new class of systems. Binary mixtures of bacteriophage viruses and (small) latex spheres with varying size ratios showed a surprisingly rich phase behaviour, including a lamellar phase 8]. In this phase, the lamellae are defined by a "smectic" arrangement of the rodlike virus particles in layers with the spherical latex particles in between the layers [8, [9]. These results are remarkable for two reasons. First, unlike the previous archetypal cases of MPS, we are dealing with a binary mixture which phase separates on a microscopic scale. There is no "hard" constraint like a chemical bond that prevents the two species from phase separating on a macroscopic scale, and both species remain in a fluid state within the layers. Second, it was argued that the virus particles as well as the latex spheres can be modeled to a good extent to interact as hard bodies. Consequently, the driving force causing this MPS must be of an entropic nature. This is also in stark contrast with MPS in block copolymers, LCP's and amphiphiles where the dependence on temperature is strong and hence indicates a predominantly energetic effect. The possibility of this type of MPS was already explored in computer simulations [10] and found to be qualitatively well described within the so-called second virial approximation 10, 11], the validity of which can only be guaranteed at low densities. However, as the experimental systems are far from dilute this last treatment may not capture all the essential ingredients. It has been argued that MPS in binary mixtures may be caused by 
the so-called depletion effect [8, 9 ], which is generically a many-body interaction effect and is not well described with a second-virial theory. Consequently, a more accurate approach would be required, certainly in order to resolve in detail what prevents the system from demixing macroscopically.

That entropy per se can be the driving force for phase transitions has by now been well established. There are many examples ranging from ordering in monodisperse systems like the liquid-to-crystal transition in hard spheres [12] and the isotropic-to-nematic transition in slender hard rods 13], to demixing in binary mixtures, like e.g. the Asakura-Oosawa (AO) mixture of hard spheres and ideal spheres, which is used as a model for colloid-polymer mixtures [14, 15, 16]. In essence, the physical mechanism in all these systems is the same; the gain in effective "free volume" available to the particles upon ordering offsets the loss of entropy of disorder or mixing respectively. For the $\mathrm{AO}$ mixture this is usually referred to as the previously mentioned depletion effect; the ideal polymers are depleted from a shell around the impenetrable colloids. Overlap of these depletion shells increases the free volume available to the polymers and hence this system phase separates into a colloid-rich and a colloid-poor fluid [17]. However, whereas entropy can induce phase behaviour that is at least as rich as seen in energetic systems, MPS remains a very rare phenomenon in entropic systems [18].

A variant of the depletion effect was also recently discovered in theoretical treatments of binary mixtures of thin and thick hard rods [19, 20]. These systems are seen to be able to phase separate in two isotropic fluid phases due to depletion. Here, however, the depletion interaction appears as a genuine two-body effect [20], in contrast to the AO system, in which it is predominantly a three-body effect. Consequently, this form of depletion effect survives the Onsager limit (length $\gg$ width) applied to both species of rods, and for sufficiently asymmetric rods, preempts the usual transition to the orientationally ordered nematic phase [19, 20, 21, 22]. These predictions have since been corroborated by simulations [23, 24]. In the present paper, we propose to use the two-body depletion effects between slender rods of different diameters to construct a system which shows entropy-induced MPS. Taking our cue from the concepts developed in the field of thermotropic block copolymers, we connect a chain of freely rotating "thick" hard rods to a chain of freely rotating "thin" hard rods. The above-mentioned unfavourable depletion interaction between these two types of rods provides the tendency to fully demix, whereas the joint (connecting the two strands) prevents this. The soconstructed system of freely jointed hard diblock copolymers (HDC) is in our view one of the most simple systems conceivable showing entropy-induced MPS. Furthermore, and contrary to the case of MPS in the binary rod-sphere mixtures, the physical mechanism is both clear and robust. Of course, there is as yet no direct candidate for an experimental system well described. However, it may certainly be possible for experimentalists to connect (possibly long and flexible) chemically inert polymers to the ends of virus particles like TMV 25, 26]. Together with an appropriate solvent this may mimick an effective rodcoil system with only hard body interactions. In this system, the polymer tails are likely to stabilize the smectic phase of the virus particles and this could be viewed as a microseparated phase.

In order to describe this system we employ a density functional theory in the second-virial or Onsager approximation starting from first principles. We assume that multiple overlaps between two chains as well as selfoverlaps of the chains are unimportant. All three of the above approximations, common in theoretical treatments of LCPs 27, 28, 29, 30], should become exact in the Onsager limit where the lengths of the rods involved is much larger than their widths. The stationarity equations that determine the stable phases in our theory are solved locally by means of a bifurcation (or, equivalently, linear stability) analysis of the isotropic fluid phase [31, 32]. Apart from fluctuations with a nonzero wave vector corresponding to a microseparated phase, we also consider spatially homogeneous fluctuations with nematic symmetry, in order to study the competition between these two types of ordering. For both phases, we obtain closed analytical expressions for the spinodal density. We find that for long chains and nonzero difference in the widths, the microseparated phase always preempts the nematic.

Naturally we want to make contact with the vast amount of literature on thermotropic block copolymers in the weak segregation limit. Most of these follow the original treatment proposed in the seminal paper by Leibler 33. Leibler considered diblock copolymers interacting via the heuristic Flory parameter $\chi$ and constructed a Landau expansion in the average composition fluctuations. By applying the "random phase approximation" and retaining only leading orders of the Fourier modes, he was able to map out more or less the complete phase diagram. Subsequent refinements extended the theory to the strong segregation regime 34], added fluctuations [35] and included extra phases [36], but did not change the essence of the approach. Leibler's results have been confirmed qualitatively by experiments (Ref. [1] and Refs. therein) and, for finite chains lengths [35], by simulations (Refs. 37, 38 and Refs. therein). The Leibler approach treats the correlations within the polymers on the Gaussian level 11. We can therefore connect to this approach by applying the Gaussian limit to our model of freely jointed HDC's. Within this limit our theory becomes equivalent to that of Leibler as far as the treatment of the intrachain interactions is concerned. However, the interchain interactions between the polymers are essentially different in the present case, as they are of a geometric nature, i.e. totally fixed by the dimensions of the composing hard rods. In the Leibler theory, these interactions are described generically by means of the freely adjustable Flory parameter. A full exploration of the parallels between the two approaches, however, was 
beyond the scope of this work.

Another class of systems, that appears as a special case of our model are the well-studied rod-coil diblock copolymers. These consist of one stiff (rodlike) block and a much more flexible part. In such systems, liquid crystalline ordering competes with MPS and a number of theoretical studies have been devoted to the subject. Most of these combine the Leibler approach with an additional Maier-Saupe anisotropic orientational interaction resulting in the appearance of a nematic phase (and sometimes an additional smectic phase) in the phase diagram, besides the various microseparated phases [39, 40, 41, 42, 43, 44, 45]. However, the ratio of the Flory and the Maier-Saupe interaction parameters in these approaches is rather arbitrary, whereas in the present approach microseparated and nematic ordering both result from the same geometric origin with no room for additional tuning.

Finally, there has been some related work on more idealized but conceptually simpler systems in the context of entropic liquid crystals. Hołyst considered parallel nailshaped particles which showed a nematic-to-smectic $\mathrm{A}_{\mathrm{d}}$ transition [46]. As a model for surfactants, Bolhuis and Frenkel studied non-additive complexes of hard spheres and ideal spherocylinder-tails 47] where Schmidt and von Ferber used hard slender rods for the tails 48 Of particular relevance to the present work is Ref. [4] where Düchs and Sullivan investigate the phase behavior of hard wormlike diblock copolymers. However, in this latter work only differences in persistence length are considered and not in thickness between the two components. Consequently they only find competition between a nematic and a (orientationally ordered) smectic phase, instead of the (orientationally disordered) lamellar phase. Moreover, only numerical solutions to the stationarity equations are presented, whereas we are able to obtain additional analytical insight through the stability analysis of the isotropic fluid phase. Lastly, van Duijneveldt and Allen used Monte Carlo simulations to study the effect of flexible tails on the phase behavior of spherocylinders [50]. This was later extended by Casey and Harrowell to rod-coil molecules of which the isolated rods do not posses a smectic phase [51].

Although our theory is formulated for chains with a finite number of rodlike segments, we devote the major part of this paper to chains with an infinite number of segments in which the correlations between the segments are Gaussian. We formulate a consistent Gaussian limit, in which the number of model parameters reduces to just three. The limit is chosen in such a way that we can still consider the competition between MPS and nematic ordering. This is achieved by letting the difference in thickness between the two types of rods to become infinitesimally small. The limit moreover is such that most of the assumptions in the original derivation of the model are fully satisfied. The most prominent results are phase diagrams as a function of the model parameters, showing the regions of stability of the microseparated or nematic

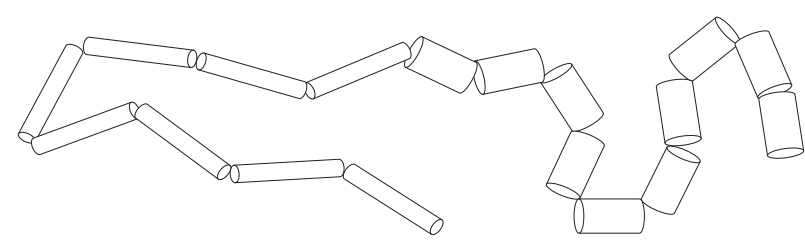

FIG. 1: An example of a hard diblock copolymer. A freelyjointed chain of $M_{\mathrm{A}}$ hard rods with dimensions $l_{\mathrm{A}}$ and $d_{\mathrm{A}}$ (left side) are connected to a freely-jointed chain of $M_{\mathrm{B}}$ hard rods with dimensions $l_{\mathrm{B}}$ and $d_{\mathrm{B}}$ (right side).

phases. Furthermore, exploiting the features of the bifurcation analysis, we are able to calculate the relative order along the polymer in the microseparated phase at the bifurcation point. The outline of the paper is as follows: in Sec. II we define the model and develop the formalism. In Sec. III we briefly discuss the symmetry of the phases involved. The bifurcation analysis is the topic of Sec. IV and the Gaussian limit is applied in Sec. V. Sec. VI is the results section and we end with a discussion in Sec. VII.

\section{MODEL AND FORMALISM}

We consider a monodisperse fluid of $N$ diblock copolymers in a volume $V$. Each polymer is a chain of freelyjointed cylindrical rods connected end-to-end where the first $M_{\mathrm{A}}$ rods are of type $\tau=A$ having length $l_{\mathrm{A}}$ and width $d_{\mathrm{A}}$ and the last $M_{\mathrm{B}}$ rods are of type $\mathrm{B}$ with dimensions $l_{\mathrm{B}}$ and $d_{\mathrm{B}}$ (see Fig. 11). We assume that both types of rods are very slender, $l_{\tau} \gg d_{\tau}$, with $\tau \in\{\mathrm{A}, \mathrm{B}\}$, hard bodies, i.e. impenetrable to other rods. The total number of segments in a chain is $M=M_{\mathrm{A}}+M_{\mathrm{B}}$ and every segment has a label $m \in\{1, \ldots, M\}$ specifying its position in the chain. The state of a segment is described by the position $\mathbf{r}_{m}$ of its center of mass and an orientation, being a unit vector $\hat{\omega}_{m}$ pointing along its long axis in the direction of increasing $m$. The configuration of a whole chain $\boldsymbol{\xi}$ is fully characterized by the position of one of its segments (say the first; $\mathbf{r}_{1}$ ) and the orientations of all of them, $\boldsymbol{\Omega}=\left\{\hat{\omega}_{1}, \ldots, \hat{\omega}_{M}\right\}$, so $\boldsymbol{\xi}=\left\{\mathbf{r}_{1}, \boldsymbol{\Omega}\right\}$. The position of a segment $m$ is then given by $\mathbf{r}_{m}=\mathbf{r}_{1}+\frac{1}{2} \sum_{k=1}^{m-1}\left(l_{k} \hat{\omega}_{k}+l_{k+1} \hat{\omega}_{k+1}\right)$ where $l_{k}=l_{\mathrm{A}}$ if $k \leq M_{\mathrm{A}}$ and $l_{\mathrm{B}}$ if $k \geq M_{\mathrm{A}}+1$.

In density functional theory (DFT) the free energy of a (possibly inhomogeneous) fluid of molecules is expressed as a functional of the single-molecule configuration distribution function, $\rho^{(1)}(\boldsymbol{\xi})$ [52]. Using the second-virial (or Onsager) approximation it is formulated as follows 53.

$$
\begin{aligned}
\beta \mathcal{F}\left[\rho^{(1)}\right]= & \int d \boldsymbol{\xi} \rho^{(1)}(\boldsymbol{\xi})\left[\log \left(\mathcal{V}_{\mathrm{T}} \rho^{(1)}(\boldsymbol{\xi})\right)-1\right] \\
& -\frac{1}{2} \iint d \boldsymbol{\xi} d \boldsymbol{\xi}^{\prime} \rho^{(1)}(\boldsymbol{\xi}) \rho^{(1)}\left(\boldsymbol{\xi}^{\prime}\right) \Phi\left(\boldsymbol{\xi}, \boldsymbol{\xi}^{\prime}\right)
\end{aligned}
$$

The integrals are over single-molecule configuration space where $\int d \boldsymbol{\xi}=\int d \mathbf{r}_{0} d \boldsymbol{\Omega}$ and $\int d \boldsymbol{\Omega}=\int \prod_{m} d \hat{\omega}_{m}$ and 
$\int d \hat{\omega}=\int_{0}^{2 \pi} d \phi \int_{0}^{\pi} d \theta \sin \theta$. Further, $\rho^{(1)}(\boldsymbol{\xi})$ is normalized as follows $\int \rho^{(1)}(\boldsymbol{\xi}) d \boldsymbol{\xi}=N$. The factor $\beta$ equals $\left(k_{B} T\right)^{-1}$ in which $k_{B}$ is Boltzmann's constant and $T$ the temperature. The volume $\mathcal{V}_{\mathrm{T}}$ we call the 'thermal volume' and is a product of the de Broglie thermal wavelengths of the molecules [53, 54]. The quantity $\Phi\left(\boldsymbol{\xi}, \boldsymbol{\xi}^{\prime}\right)$ is the Mayer function of two molecules with configurations $\boldsymbol{\xi}$ and $\boldsymbol{\xi}^{\prime}$. As we are dealing with hard segments, the potential energy $v\left(\boldsymbol{\xi}, \boldsymbol{\xi}^{\prime}\right)$ between two chains is $\infty$ when they overlap and 0 when they don't. Consequently, the Mayer function is given by

$$
\Phi\left(\boldsymbol{\xi}, \boldsymbol{\xi}^{\prime}\right)=\exp \left(-\beta v\left(\boldsymbol{\xi}, \boldsymbol{\xi}^{\prime}\right)\right)-1=\left\{\begin{array}{rl}
-1 & \text { if overlap } \\
0 & \text { if no overlap }
\end{array} .\right.
$$

The configurations of both chains involved can be highly irregular and the dependence of $\Phi$ very complicated. Therefore we approximate the chain-chain Mayer function $\Phi$ by the sum of all the segment-segment Mayer functions $\phi_{m, m^{\prime}}$,

$$
\Phi\left(\boldsymbol{\xi}, \boldsymbol{\xi}^{\prime}\right)=\sum_{m, m^{\prime}=1}^{M} \phi_{m, m^{\prime}}\left(\mathbf{r}_{m}-\mathbf{r}_{m^{\prime}}, \hat{\omega}_{m}, \hat{\omega}_{m^{\prime}}\right) .
$$

This expression, to which only individual pairs of segments contribute, is actually the first term in a systematic expansion of the Mayer function. Higher order terms involve interactions between more than two segments simultaneously [30]. At this point we note that apart from (i) the second virial approximation and (ii) the above expression for the chain-chain Mayer function, another (iii) approximation has been made. In this formalism the chains are allowed to self overlap, i.e. other than the spatial constraint that successive segments are connected to each other there are no interactions within the chain. All three of these approximations are commonly used and corrections to the first two are small when $l_{\tau} \gg d_{\tau}$ [27, 28, 29]. The neglect of the effects of self-overlap is assumed to be reasonable in a dense polymer melt [55] where screening effectively compensates the intramolecular interactions and as a result interactions between distant parts of the same chain are indistinguishable from interactions with the average environment because of loss of intrachain correlations.

In thermodynamic equilibrium, the free energy reaches a minimum and the functional is stationary. Therefore, we consider the variation of Eq. 1 with respect to $\rho^{(1)}$,

$$
\frac{\delta}{\delta \rho^{(1)}(\boldsymbol{\xi})} \beta \mathcal{F}-\beta \mu=0
$$

with the chemical potential $\mu$ playing the role of Lagrange multiplier needed to enforce normalization. Eliminating $\mu$ from Eq. 4 yields the (selfconsistent) stationarity equation,

$$
\rho^{(1)}(\boldsymbol{\xi})=\frac{N \exp \left[\int d \boldsymbol{\xi}^{\prime} \rho^{(1)}\left(\boldsymbol{\xi}^{\prime}\right) \Phi_{M}\left(\boldsymbol{\xi}, \boldsymbol{\xi}^{\prime}\right)\right]}{\int d \boldsymbol{\xi} \exp \left[\int d \boldsymbol{\xi}^{\prime} \rho^{(1)}\left(\boldsymbol{\xi}^{\prime}\right) \Phi_{M}\left(\boldsymbol{\xi}, \boldsymbol{\xi}^{\prime}\right)\right]},
$$

In order to proceed, we define the single-segment distribution function (SDF) (of segment $m$ ), $\rho_{m}\left(\mathbf{r}_{m}, \hat{\omega}_{m}\right)$, in the following way

$$
\begin{aligned}
\rho_{m}\left(\mathbf{r}_{m}, \hat{\omega}_{m}\right)=\int & \prod_{k \neq m} d \hat{\omega}_{k} \rho^{(1)}(\boldsymbol{\xi}) \\
& =\int \prod_{k \neq m} d \hat{\omega}_{k} \rho^{(1)}\left(\mathbf{r}_{1}\left(\mathbf{r}_{m}, \boldsymbol{\Omega}\right), \boldsymbol{\Omega}\right),
\end{aligned}
$$

in which $\mathbf{r}_{1}$ is given by $\mathbf{r}_{m}-\frac{1}{2} \sum_{k=1}^{m-1}\left(l_{k} \hat{\omega}_{k}+l_{k+1} \hat{\omega}_{k+1}\right)$ and the product is over all segments $k$ but the $m$ th. Integrating Eq. [5 over all $\hat{\omega}_{k}$ except for $\hat{\omega}_{m}$ as well and using Eq. 3 we obtain a set of equations,

$$
\begin{aligned}
& \rho_{m}\left(\mathbf{r}_{m}, \hat{\omega}_{m}\right)=\frac{N}{Q} \int \prod_{k \neq m} d \hat{\omega}_{k} \times \\
& \exp \left[\sum_{k, k^{\prime}=1}^{M} \int d \mathbf{r}_{k^{\prime}}^{\prime} d \hat{\omega}_{k^{\prime}}^{\prime} \rho_{k^{\prime}}\left(\mathbf{r}_{k^{\prime}}^{\prime}, \hat{\omega}_{k^{\prime}}^{\prime}\right) \phi_{k, k^{\prime}}\left(\mathbf{r}_{k}-\mathbf{r}_{k^{\prime}}^{\prime}, \hat{\omega}_{k}, \hat{\omega}_{k^{\prime}}^{\prime}\right)\right],
\end{aligned}
$$

where $Q$ is the normalization factor; i.e. the SDF's are normalized in the same way as $\rho^{(1)}$ : i.e. $\int d \mathbf{r} d \hat{\omega} \rho_{m}(\mathbf{r}, \hat{\omega})=$ $N$.

\section{PHASE BEHAVIOUR AND ORDER PARAMETERS}

\section{A. Isotropic Phase}

At low polymer number density, $n=N / V$, the system is in the isotropic fluid phase, and $\rho_{m}\left(\mathbf{r}_{m}, \hat{\omega}_{m}\right)$ is a constant, so due to normalization, $\rho_{m}^{\text {(iso) }}=n / 4 \pi$. Consequently,

$$
\begin{aligned}
\int d \mathbf{r}^{\prime} d \hat{\omega}^{\prime} \rho_{m}^{\text {(iso) }} \phi_{k, k^{\prime}}\left(\mathbf{r}_{k}-\mathbf{r}^{\prime}, \hat{\omega}_{k}, \hat{\omega}^{\prime}\right) \\
=-\frac{n}{4 \pi} \int d \hat{\omega}^{\prime} l_{k} l_{k^{\prime}}\left(d_{k}+d_{k^{\prime}}\right) \sin \gamma\left(\hat{\omega}_{k} \cdot \hat{\omega}^{\prime}\right) \\
=-\frac{1}{4} \pi n l_{k} l_{k^{\prime}}\left(d_{k}+d_{k^{\prime}}\right),
\end{aligned}
$$

where $\gamma\left(\hat{\omega} \cdot \hat{\omega}^{\prime}\right)$ is the planar angle between $\hat{\omega}$ and $\hat{\omega}^{\prime}$ and one can recognize $l_{k} l_{k^{\prime}}\left(d_{k}+d_{k^{\prime}}\right) \sin \gamma\left(\hat{\omega}, \hat{\omega}^{\prime}\right)$ as the excluded volume of two rods $k$ and $k^{\prime}$ with respective orientations $\hat{\omega}$ and $\hat{\omega}^{\prime}$. This yields the following normalization factor in the isotropic phase

$$
\begin{aligned}
& \quad Q_{\text {iso }}=(4 \pi)^{M_{\mathrm{A}}+M_{\mathrm{B}}} V \times \\
& \exp \left[-\frac{1}{2} \pi n\left(M_{\mathrm{A}}^{2} l_{\mathrm{A}}^{2} d_{\mathrm{A}}+M_{\mathrm{A}} M_{\mathrm{B}} l_{\mathrm{A}} l_{\mathrm{B}}\left(d_{\mathrm{A}}+d_{\mathrm{B}}\right)+M_{\mathrm{B}}^{2} l_{\mathrm{B}}^{2} d_{\mathrm{B}}\right)\right]
\end{aligned}
$$

Choosing the dimensions of rod $\mathrm{A}$ as units, we define

$$
\tilde{l}=l_{\mathrm{B}} / l_{\mathrm{A}}, \tilde{d}=d_{\mathrm{B}} / d_{\mathrm{A}}, \quad \tilde{M}=M_{\mathrm{B}} / M_{\mathrm{A}},
$$


and a dimensionless segment density in a symmetric way,

$$
\tilde{n}=2 n\left(M_{\mathrm{A}} l_{\mathrm{A}}^{2} d_{\mathrm{A}}+M_{\mathrm{B}} l_{\mathrm{B}}^{2} d_{\mathrm{B}}\right) .
$$

Then, Eq. 9 becomes

$$
Q_{\text {iso }}=(4 \pi)^{M} V \exp \left[-\frac{\pi}{4} \tilde{n} M \frac{(1+\tilde{M} \tilde{l})(1+\tilde{M} \tilde{l} \tilde{d})}{(1+\tilde{M})\left(1+\tilde{M} \tilde{l}^{2} \tilde{d}\right)}\right]
$$

where we have also used $M=M_{\mathrm{A}}+M_{\mathrm{B}}$. We also note that the normalization factor $Q_{\text {iso }}$ is exactly the partition sum of the block copolymers in the isotropic phase.

\section{B. Nematic Phase}

In the (uniaxial) nematic phase, there is orientational order with respect to a direction $\hat{n}$, however, the system is still spatially homogeneous. Therefore, the SDF can be expanded in Legendre polynomials.

$$
\rho_{m}(\mathbf{r}, \hat{\omega})=\rho_{m}(\hat{\omega})=n \sum_{j=0}^{\infty} \frac{2 j+1}{4 \pi} a_{m}^{(j)} P_{j}(\hat{\omega} \cdot \hat{n}),
$$

with coefficients

$$
n a_{m}^{(j)}=\int d \hat{\omega}^{\prime} P_{j}\left(\hat{\omega}^{\prime} \cdot \hat{n}\right) \rho_{m}\left(\hat{\omega}^{\prime}\right)
$$

Due to normalization, $a_{m}^{(0)}=1$ as it is in the isotropic phase and due to up-down symmetry of the nematic, all $a_{m}^{(j)}=0$ for odd $j$ (in the isotropic fluid, $a_{m}^{(j)}=0$ for all $j \neq 0$ ). The lowest-order coefficient different in the nematic and the isotropic phase is $a_{m}^{(2)}$ which is the usual Maier-Saupe order parameter. The physical incentive to form a nematic is that the average excluded volume between rods is smaller (and therefore the average free volume available to the rods is larger) in the nematic phase [13].

\section{Microseparated Phase}

Microseparated phases consist of spatially distributed regions rich either in type-A or type-B rods and are typically governed by a single dominant wavelength. These phases exist in a variety of types exhibiting various degrees of symmetry, e.g. lamellar, hexagonal, bcc and even more exotic morphologies like the gyroid phase [1, 36]. In this paper we do not consider the various symmetries of microseparated phases but focus on the magnitude of the dominant wavelength and the density for which it becomes unstable. To that end, we observe that the SDF can be expanded in terms of plane waves

$$
\rho_{m}(\mathbf{r}, \hat{\omega})=\sum_{\mathbf{q} \in \mathbb{L}} \hat{\rho}_{m}(\mathbf{q}, \hat{\omega}) \mathrm{e}^{i \mathbf{q} \cdot \mathbf{r}}
$$

with $\mathbb{L}$ some set of wave vectors and the "coefficients" given by

$$
\hat{\rho}_{m}(\mathbf{q}, \hat{\omega})=V^{-1} \int d \mathbf{r}^{\prime} \mathrm{e}^{-i \mathbf{q} \cdot \mathbf{r}^{\prime}} \rho_{m}\left(\mathbf{r}^{\prime}, \hat{\omega}\right) .
$$

In general there will be orientational order within the domains and consequently the coefficients still depend on the orientation. If needed, one could proceed and expand these coefficients again in spherical harmonics. However, in order to simplify the analysis, this additional orientational order in the microseparated phase is usually neglected which we will show in Sec. $\nabla$ is permitted for the case of infinitely long polymers. In homogeneous fluid phases like the nematic, the SDF is independent on the spatial coordinate and only the coefficient $\hat{\rho}_{m}(\mathbf{0}, \hat{\omega})$ at zero wavenumber survives.

\section{BIFURCATION ANALYSIS}

\section{A. The Bifurcation Equation}

At low densities, the isotropic phase is the globally stable phase, but at higher densities it will become unstable with respect to lower symmetry phases exhibiting some form of ordering. Points where these lower-symmetry solutions branch off the isotropic solution are called bifurcation points and the densities at which this happens, bifurcation densities. Different solutions may bifurcate at different densities from the isotropic phase. Generically the particular solution which bifurcates at the lowest density, will give rise to the first ordered phase that is also thermodynamically stable with respect to the isotropic phase. In this section, we perform a linear stability (or bifurcation) analysis around the isotropic parent solution, along the lines of Refs. 30, 31, 32. Consequently, we assume isotropic distributions with a perturbation of lower symmetry,

$$
\rho_{m}(\mathbf{r}, \hat{\omega})=\frac{n}{4 \pi}+\varepsilon \rho_{m, 1}(\mathbf{r}, \hat{\omega})
$$

where the proper normalisation of the SDF requires $\int d \mathbf{r} d \hat{\omega} \rho_{m, 1}(\mathbf{r}, \hat{\omega})=0$. Inserting this in the stationarity equations 7 we linearize the exponent with respect to the infinitesimal parameter $\varepsilon$,

$$
\begin{gathered}
\exp \left[\sum_{k, k^{\prime}=1}^{M} \int d \mathbf{r}^{\prime} d \hat{\omega}^{\prime} \rho_{k^{\prime}}\left(\mathbf{r}^{\prime}, \hat{\omega}^{\prime}\right) \phi_{k, k^{\prime}}\left(\mathbf{r}_{k}-\mathbf{r}^{\prime}, \hat{\omega}_{k}, \hat{\omega}^{\prime}\right)\right]= \\
\exp \left[-\frac{1}{4} \pi n \sum_{k, k^{\prime}=1}^{M} l_{k} l_{k^{\prime}}\left(d_{k}+d_{k^{\prime}}\right)\right] \times \\
\left(1+\varepsilon \sum_{k, k^{\prime}} \int d \mathbf{r}^{\prime} d \hat{\omega}^{\prime} \rho_{k^{\prime}, 1}\left(\mathbf{r}^{\prime}, \hat{\omega}^{\prime}\right) \phi_{k, k^{\prime}}\left(\mathbf{r}_{k}-\mathbf{r}^{\prime}, \hat{\omega}_{k}, \hat{\omega}^{\prime}\right)\right) .
\end{gathered}
$$


Equating orders in $\varepsilon$, to zeroth order, we re-obtain the isotropic result, Eq. 9] To first order this yields the socalled bifurcation equations,

$$
\begin{aligned}
& \rho_{m, 1}\left(\mathbf{r}_{m}, \hat{\omega}_{m}\right)=\frac{n}{(4 \pi)^{M}} \int \prod_{k^{\prime \prime} \neq m} d \hat{\omega}_{k^{\prime \prime}} \times \\
& \sum_{k, k^{\prime}} \int d \mathbf{r}^{\prime} d \hat{\omega}^{\prime} \rho_{k^{\prime}, 1}\left(\mathbf{r}^{\prime}, \hat{\omega}^{\prime}\right) \phi_{k, k^{\prime}}\left(\mathbf{r}_{k}-\mathbf{r}^{\prime}, \hat{\omega}_{k}, \hat{\omega}^{\prime}\right) .
\end{aligned}
$$

These can be interpreted a generalized linear eigenvalue problem with eigenfunctions $\rho_{m, 1}(\mathbf{r}, \hat{\omega})$ and eigenvalue $n$, the bifurcation density. There is an infinite hierarchy of solutions to Eq. 19 for varying degrees of symmetry. However, we are only interested in the one (or the few) corresponding to the lowest bifurcation density. Note that the explicit dependence on the normalization factor $Q$ has dropped out since integration over $\mathbf{r}_{m}$ and $\hat{\omega}_{m}$ trivially yields zero on the left hand side by definition and, after rearrangement of the integrals made possible by the finite range of the Mayer functions $\phi_{k, k^{\prime}}$, also on the right hand side.

In order to make the bifurcation equation, Eq.19] more transparent we define for the moment as an auxiliary quantity, the fields,

$$
H_{k}\left(\mathbf{r}_{k}, \hat{\omega}_{k}\right)=\sum_{k^{\prime}} \int d \mathbf{r}^{\prime} d \hat{\omega}^{\prime} \rho_{k^{\prime}, 1}\left(\mathbf{r}^{\prime}, \hat{\omega}^{\prime}\right) \phi_{k, k^{\prime}}\left(\mathbf{r}_{k}-\mathbf{r}^{\prime}, \hat{\omega}_{k}, \hat{\omega}^{\prime}\right) .
$$

in terms of which the bifurcation equation becomes

$$
\rho_{m, 1}\left(\mathbf{r}_{m}, \hat{\omega}_{m}\right)=\frac{n}{(4 \pi)^{M}} \sum_{k} \int \prod_{k^{\prime \prime} \neq m} d \hat{\omega}_{k^{\prime \prime}} H_{k}\left(\mathbf{r}_{k}, \hat{\omega}_{k}\right)
$$

However, this field $H_{k}$ is a function of $\mathbf{r}_{k}$ and $\hat{\omega}_{k}$ whereas on the left of Eq. 21] we have a function of $\mathbf{r}_{m}$ and $\hat{\omega}_{m}$. And these are not independent and as

$$
\mathbf{r}_{m}=\mathbf{r}_{k}+\mathcal{P}_{k, m}
$$

where the vectorial "path" $\mathcal{P}_{k, m}$ between $k$ and $m$ is given by

$$
\mathcal{P}_{k, m}=\frac{1}{2} \sum_{k^{\prime}=k}^{m-1}\left(l_{k^{\prime}} \hat{\omega}_{k^{\prime}}+l_{k^{\prime}+1} \hat{\omega}_{k^{\prime}+1}\right),
$$

for $k<m$. Further, $\mathcal{P}_{m, m}=0$ and the case of $k>m$ can be obtained by realizing that $\mathcal{P}_{k, m}=-\mathcal{P}_{m, k}$. Consequently, the interlying orientional integrations in Eq. 21 have to make the connection and "transfer" the field from segments $k$ to $m$.

We return to Eq. 19] and insert the constraint, Eq. 22 via a delta function

$$
\begin{aligned}
& \rho_{m, 1}\left(\mathbf{r}_{m}, \hat{\omega}_{m}\right)= \\
& \frac{n}{(4 \pi)^{M}} \sum_{k} \int \prod_{k^{\prime \prime} \neq m} d \hat{\omega}_{k^{\prime \prime}} \int d \mathbf{r}_{k} \delta\left(\mathbf{r}_{m}-\mathbf{r}_{k}+\mathcal{P}_{m, k}\right) \times \\
& \sum_{k^{\prime}} \int d \mathbf{r}^{\prime} d \hat{\omega}^{\prime} \rho_{k^{\prime}, 1}\left(\mathbf{r}^{\prime}, \hat{\omega}^{\prime}\right) \phi_{k, k^{\prime}}\left(\mathbf{r}_{k}-\mathbf{r}^{\prime}, \hat{\omega}_{k}, \hat{\omega}^{\prime}\right) .
\end{aligned}
$$

Next, we observe that in Eq24 there appear two spatial convolution integrals. Therefore, it makes sense to proceed with a Fourier transform (i.e. $\hat{g}(\mathbf{q})=$ $\left.V^{-1} \int d \mathbf{r}_{m} \mathrm{e}^{-i \mathbf{q} \cdot \mathbf{r}_{m}} g\left(\mathbf{r}_{m}\right)\right)$, yielding

$$
\begin{gathered}
\hat{\rho}_{m, 1}\left(\mathbf{q}, \hat{\omega}_{m}\right)=\frac{n}{(4 \pi)^{M}} \sum_{k} \int \prod_{k^{\prime \prime} \neq m} d \hat{\omega}_{k^{\prime \prime}} \mathrm{e}^{-i \mathbf{q} \cdot \mathcal{P}_{k, m}} \times \\
\sum_{k^{\prime}} \int d \hat{\omega}^{\prime} \hat{\rho}_{k^{\prime}, 1}\left(\mathbf{q}, \hat{\omega}^{\prime}\right) \hat{\phi}_{k, k^{\prime}}\left(\mathbf{q}, \hat{\omega}_{k}, \hat{\omega}^{\prime}\right) .
\end{gathered}
$$

This is the general form of the bifurcation equation for a lower-symmetry solution bifurcating off the isotropicfluid parent solution. Note that the $\mathbf{q}$-vector is the same for all segments. Furthermore, at this point, we have not yet specified the internal structure of the polymer, only that it is a chain of cylindrically-symmetric (rodlike) segments which contains no closed loops. Concerning the rodlike segments, the Fourier transformed Mayer function $\hat{\phi}_{k, k^{\prime}}$ is calculated in Appendix $₫$ and is for very slender segments $\left(l_{k} \gg d_{k}\right)$ given by,

$$
\begin{aligned}
\hat{\phi}_{k, k^{\prime}}\left(\mathbf{q}, \hat{\omega}_{k}, \hat{\omega}_{k^{\prime}}\right)= & -l_{k} l_{k^{\prime}}\left(d_{k}+d_{k^{\prime}}\right)\left|\hat{\omega}_{k} \times \hat{\omega}_{k^{\prime}}^{\prime}\right| \times \\
& j_{0}\left(\frac{1}{2} l_{k} \mathbf{q} \cdot \hat{\omega}_{k}\right) j_{0}\left(\frac{1}{2} l_{k^{\prime}} \mathbf{q} \cdot \hat{\omega}_{k^{\prime}}\right),
\end{aligned}
$$

where we have already discarded higher-order terms containing $\left(d_{k}+d_{k^{\prime}}\right) \mathbf{q}$ as the wave vector will be at most of order $1 / l_{\mathrm{A}, \mathrm{B}}$ so these terms will be small. The function $j_{0}(x)=\sin x / x$ is the spherical Bessel function of zeroth order. We proceed by solving Eq. 25] to which we refer as the bifurcation equation from now on.

\section{B. Nematic Solution}

We first consider the nematic solution, which is also the simplest. In the nematic phase, $\mathbf{q}=0$ and the orientational integrals in the bifurcation equation are trivial and it reduces to

$$
\hat{\rho}_{m, 1}\left(\hat{\omega}_{m}\right)=\frac{n}{4 \pi} \sum_{k^{\prime}} \int d \hat{\omega}^{\prime} \hat{\rho}_{k^{\prime}, 1}\left(\hat{\omega}^{\prime}\right) \hat{\phi}_{m, k^{\prime}}\left(\hat{\omega}_{m}, \hat{\omega}^{\prime}\right),
$$

where $\hat{\rho}_{m, 1}\left(\hat{\omega}_{m}\right)=\hat{\rho}_{m, 1}\left(\mathbf{0}, \hat{\omega}_{m}\right)$ and

$$
\hat{\phi}_{m, k^{\prime}}\left(\hat{\omega}_{m}, \hat{\omega}_{k^{\prime}}\right)=-l_{m} l_{k^{\prime}}\left(d_{m}+d_{k^{\prime}}\right)\left|\hat{\omega}_{m} \times \hat{\omega}_{k^{\prime}}^{\prime}\right| .
$$

is simply minus the excluded volume of two rods with fixed orientations, $\hat{\omega}_{m}$ and $\hat{\omega}_{k^{\prime}}$. This bifurcation equation is the same as that of a mixture disconnected rods [20, so for orientational ordering the connectivity of the rods within the chains does not play a role. The kernel $\hat{\phi}_{m, k^{\prime}}$ is now only a function of the planar angle $\gamma$ between the orientations of the rods, $\left|\hat{\omega}_{m} \times \hat{\omega}_{k^{\prime}}^{\prime}\right|=\mid \sin \gamma\left(\hat{\omega}_{m}\right.$. $\left.\hat{\omega}_{k^{\prime}}\right) \mid$. Consequently, due to this uniaxial symmetry the eigenfunctions of $\hat{\phi}_{m, k^{\prime}}$ and therefore of Eq. 27 are simply the Legendre polynomials $P_{j}$ (see Appendix [B),

$\int d \hat{\omega}^{\prime} \hat{\phi}_{m, k^{\prime}}\left(\hat{\omega}, \hat{\omega}^{\prime}\right) P_{j}\left(\hat{\omega}^{\prime} \cdot \hat{n}\right)=-l_{m} l_{k^{\prime}}\left(d_{m}+d_{k^{\prime}}\right) s_{j} P_{j}(\hat{\omega} \cdot \hat{n})$, 
with $s_{j}$ the Legendre coefficients of $|\sin \gamma|$. In case of the nematic phase, it is well known that this becomes first unstable with respect to the mode $j=2$, so $\hat{\rho}_{m, 1}\left(\hat{\omega}_{m}\right)=$ $(5 / 4 \pi) n c_{m}^{(2)} P_{2}\left(\hat{\omega}_{m} \cdot \hat{n}\right)$ with $c_{m}^{(2)}$ the Legendre coefficients. Then, the bifurcation equation becomes

$$
c_{m}^{(2)}=-\frac{n}{4 \pi} \sum_{k^{\prime}} l_{m} l_{k^{\prime}}\left(d_{m}+d_{k^{\prime}}\right) s_{2} c_{k^{\prime}}^{(2)}
$$

with $s_{2}=-\pi^{2} / 8$. This is an $M \times M$ matrix eigenvalue equation and therefore in principle much too large to solve. However, by observing that the geometric factor on the right hand side does not so much depend on the segments $m, k^{\prime}$ but on whether they belong to A or $\mathrm{B}$, we can split the summation, $\sum_{k^{\prime}}=\sum_{\tau} \sum_{k^{\prime} \in \tau}$ with $\tau=\mathrm{A}, \mathrm{B}$. Then, we can define the "type-average" coefficients, $c_{\tau}^{(2)}=\left(1 / M_{\tau}\right) \sum_{m \in \tau} c_{m}^{(2)}$ and Eq. 30] becomes,

$$
c_{\tau}^{(2)}=\frac{\pi n}{32} \sum_{\tau^{\prime}=\mathrm{A}, \mathrm{B}} M_{\tau^{\prime}} l_{\tau} l_{\tau^{\prime}}\left(d_{\tau}+d_{\tau^{\prime}}\right) c_{\tau^{\prime}}^{(2)}
$$

Rewriting this in terms of dimensionless quantities,

$$
\mathbf{c}_{2}=\frac{\pi \tilde{n}}{32\left(1+\tilde{M} \tilde{l}^{2} \tilde{d}\right)} \mathbf{G}_{2} \mathbf{c}_{2}
$$

with

$$
\mathbf{G}_{2}=\left[\begin{array}{cc}
1 & \frac{1}{2} \tilde{M} \tilde{l}(1+\tilde{d}) \\
\frac{1}{2} \tilde{l}(1+\tilde{d}) & \tilde{M} \tilde{l}^{2} \tilde{d}
\end{array}\right] \text { and } \mathbf{c}_{2}=\left(\begin{array}{c}
c_{\mathrm{A}}^{(2)} \\
c_{\mathrm{B}}^{(2)}
\end{array}\right)
$$

we now have reduced the problem to a simple $2 \times 2$ matrix eigenvalue equation. There are two solutions for the density,

$$
\begin{aligned}
\tilde{n}_{ \pm}= & \frac{32\left(1+\tilde{M} \tilde{l}^{2} \tilde{d}\right)}{\pi} \times \\
& \left(\operatorname{tr} \mathbf{G}_{2} \pm \sqrt{\operatorname{tr}^{2} \mathbf{G}_{2}-4 \operatorname{det} \mathbf{G}_{2}}\right) /\left(2 \operatorname{det} \mathbf{G}_{2}\right)
\end{aligned}
$$

with det and tr denoting the determinant and trace respectively. As the determinant of $\mathbf{G}_{2}$ is negative, only the minus sign in Eq. 34 yields a positive bifurcation density $\tilde{n}_{\text {nem }}$, so

$$
\begin{aligned}
\tilde{n}_{\mathrm{nem}} & =\frac{32\left(1+\tilde{M} \tilde{l}^{2} \tilde{d}\right)}{\pi} \times \\
& \left(\operatorname{tr} \mathbf{G}_{2}-\sqrt{\operatorname{tr}^{2} \mathbf{G}_{2}-4 \operatorname{det} \mathbf{G}_{2}}\right) /\left(2 \operatorname{det} \mathbf{G}_{2}\right)
\end{aligned}
$$

Note that, within the context of the model as introduced in Sec. III this analytic expression for the nematic bifurcation is an exact result. In the wider context of liquid crystalline polymers, a more general derivation of the nematic bifurcation density can be found in Ref. [32].

\section{Microseparated Solution}

In a microseparated phase, the wave vector $\mathbf{q}$ is nonzero and the orientational integrals in the bifurcation equation have to be performed explicitly. However, we can make much progress by observing that most of the integrals are still trivial, i.e. if segment $k^{\prime \prime}$ does not lie between $k$ and $m$ it does not help to "pass on" the infinitesimal field $H_{k}$ or equivalently, there is no dependence in the factor $\exp \left(-i \mathbf{q} \cdot \mathcal{P}_{k, m}\right)$. Consequently, these $M-|m-k|-1$ integrations each contribute a factor $\int d \hat{\omega}=4 \pi$ which is in total $(4 \pi)^{M-|m-k|-1}$. On the other hand, concerning the intermediate segments $k^{\prime \prime}$ between $k$ and $m$; the only dependence on $\hat{\omega}_{k^{\prime \prime}}$ is in the path $\mathcal{P}_{k, m}$. Therefore, suppose for a moment that $k+1<m$,

$$
\begin{gathered}
\int \prod_{k^{\prime \prime}=k+1}^{m-1} d \hat{\omega}_{k^{\prime \prime}} \mathrm{e}^{-i \mathbf{q} \cdot \mathcal{P}_{k, m}}= \\
\mathrm{e}^{-\frac{1}{2} i \mathbf{q} \cdot l_{k} \hat{\omega}_{k}}\left(\prod_{k^{\prime \prime}=k+1}^{m-1} \int d \hat{\omega} \mathrm{e}^{-i \mathbf{q} \cdot l_{k^{\prime \prime}} \hat{\omega}}\right) \mathrm{e}^{-\frac{1}{2} i \mathbf{q} \cdot l_{m} \hat{\omega}_{m}}
\end{gathered}
$$

and it is easy to show that

$$
\int d \hat{\omega} \mathrm{e}^{-i \mathbf{q} \cdot l_{k^{\prime \prime}} \hat{\omega}}=4 \pi \frac{\sin q l_{k^{\prime \prime}}}{q l_{k^{\prime \prime}}}=4 \pi j_{0}\left(q l_{k^{\prime \prime}}\right),
$$

where we have used $\mathbf{q}=q \hat{q}$ with $q$ being the length and the unit vector $\hat{q}$ the direction of the wave vector. When $m+1<k$, there is an extra minus sign as $\mathcal{P}_{m, k}=-\mathcal{P}_{k, m}$ but this does not change the result 37. only the end factors in Eq. 36] Consequently, we define the factor

$$
F_{k, m}(q)=\left\{\begin{array}{ll}
\prod_{k^{\prime \prime}=k+1}^{m-1} j_{0}\left(q l_{k^{\prime \prime}}\right) & \text { for } k<m-1 \\
1 & \text { for } k=m-1, m
\end{array},\right.
$$

which is symmetric so $F_{k, m}(q)=F_{m, k}(q)$. Inserting this in the bifurcation equation yields,

$$
\begin{aligned}
& \hat{\rho}_{m, 1}\left(\mathbf{q}, \hat{\omega}_{m}\right)= \\
& \frac{n}{4 \pi} \sum_{k^{\prime}} \int d \hat{\omega}^{\prime} \hat{\rho}_{k^{\prime}, 1}\left(\mathbf{q}, \hat{\omega}^{\prime}\right) \hat{\phi}_{m, k^{\prime}}\left(\mathbf{q}, \hat{\omega}_{m}, \hat{\omega}^{\prime}\right)+ \\
& \frac{n}{(4 \pi)^{2}} \sum_{k \neq m} \mathrm{e}^{\sigma_{m, k} \frac{1}{2} i \mathbf{q} \cdot l_{m} \hat{\omega}_{m}} F_{m, k}(q) \sum_{k^{\prime}} \times \\
& \int d \hat{\omega} d \hat{\omega}^{\prime} \cos \left(\frac{1}{2} \mathbf{q} \cdot l_{k} \hat{\omega}\right) \hat{\rho}_{k^{\prime}, 1}\left(\mathbf{q}, \hat{\omega}^{\prime}\right) \hat{\phi}_{k, k^{\prime}}\left(\mathbf{q}, \hat{\omega}, \hat{\omega}^{\prime}\right),
\end{aligned}
$$

where $\sigma_{k, m}=\operatorname{sign}(m-k)$ is the sign of $m-k$. Instead of the other "end factor" $\exp \left(\sigma_{m, k} \frac{1}{2} i \mathbf{q} \cdot l_{k} \hat{\omega}\right)$ we have used $\cos \left(\frac{1}{2} \mathbf{q} \cdot l_{k} \hat{\omega}\right)$ as within the integral only the even part in $\mathbf{q}$ survives. The first term on the right hand side is due to the infinitesimal field $H_{m}$ directly on segment $m$; the second term contains the contributions $H_{k}$ on segments $k \neq m$ which are being transferred to segment $m$ via $F_{m, k}$. At this point we note that it is impossible to solve Eq. 39] analytically for general $\mathbf{q}$ and we will introduce an approximation justified for very long polymers, 
$M_{\mathrm{A}}, M_{\mathrm{B}} \gg 1$. In this case the relevant wave vector is expected to be small in magnitude and consequently, the "end factors" as well as the wave dependence of $\hat{\phi}_{k, k^{\prime}}$ are negligible. Therefore, we replace them by their zeroth order approximations in $\mathbf{q}$,

$$
\hat{\phi}_{k, k^{\prime}}=-l_{k} l_{k^{\prime}}\left(d_{k}+d_{k^{\prime}}\right)\left|\hat{\omega}_{k} \times \hat{\omega}_{k^{\prime}}^{\prime}\right|
$$

and

$$
\begin{aligned}
& \exp \left(\sigma_{m, k} \frac{1}{2} i \mathbf{q} \cdot l_{m} \hat{\omega}_{m}\right)=1 \\
& \cos \left(\frac{1}{2} i \mathbf{q} \cdot l_{k} \hat{\omega}_{k}\right)=1
\end{aligned}
$$

Then the bifurcation equation becomes

$$
\begin{aligned}
& \hat{\rho}_{m, 1}\left(\mathbf{q}, \hat{\omega}_{m}\right)=\frac{n}{4 \pi} \sum_{k^{\prime}} \int d \hat{\omega}^{\prime} \hat{\rho}_{k^{\prime}, 1}\left(\mathbf{q}, \hat{\omega}^{\prime}\right) \hat{\phi}_{m, k^{\prime}}\left(\hat{\omega}_{m}, \hat{\omega}^{\prime}\right)+ \\
& \frac{n}{(4 \pi)^{2}} \sum_{k \neq m} F_{m, k}(q) \sum_{k^{\prime}} \int d \hat{\omega} d \hat{\omega}^{\prime} \hat{\rho}_{k^{\prime}, 1}\left(\mathbf{q}, \hat{\omega}^{\prime}\right) \hat{\phi}_{k, k^{\prime}}\left(\hat{\omega}, \hat{\omega}^{\prime}\right),
\end{aligned}
$$

where again as in the case of the nematic solutions, $\hat{\phi}_{k, k^{\prime}}\left(\hat{\omega}, \hat{\omega}^{\prime}\right)$ has the convenient property that it maps $P_{j}$ on $P_{j}$. Then the only mode for which the second term on the right hand side of Eq. 42 survives (and we have wave dependence) is for $P_{0}$. (For $j \neq 0$ we simply re-obtain the nematic bifurcation equation, Eq.30) Consequently, integrating both sides over $\hat{\omega}_{m}$, we obtain

$$
c_{m}^{(0)}(q)=-\frac{n}{4 \pi} \sum_{k} F_{m, k}(q) \sum_{k^{\prime}} l_{k} l_{k^{\prime}}\left(d_{k}+d_{k^{\prime}}\right) s_{0} c_{k^{\prime}}^{(0)}(q)
$$

where we have defined $c_{m}^{(0)}(q)=\int d \hat{\omega}_{m} \hat{\rho}_{m, 1}\left(\mathbf{q}, \hat{\omega}_{m}\right)$ and where

$$
\int d \hat{\omega} \hat{\phi}_{k, k^{\prime}}\left(\hat{\omega}, \hat{\omega}^{\prime}\right)=-l_{k} l_{k^{\prime}}\left(d_{k}+d_{k^{\prime}}\right) s_{0}
$$

with $s_{0}=\pi^{2}$. The rest of the analysis is similar to the nematic case: again we have an $M \times M$ eigenvalue equation and we make use of the property of the geometric factor that it depends on the types involved and not on the segment labels, hence $\sum_{k^{\prime}}=\sum_{\tau^{\prime}} \sum_{k^{\prime} \in \tau^{\prime}}$ with $\tau^{\prime}=\mathrm{A}$, B. Defining $c_{\tau}^{(0)}(q)=\left(1 / M_{\tau}\right) \sum_{k \in \tau} c_{k}^{(0)}(q)$ and $F_{\tau, \tau^{\prime}}=\left(1 / M_{\tau} M_{\tau^{\prime}}\right) \sum_{m \in \tau} \sum_{k^{\prime} \in \tau^{\prime}} F_{m, k}$, Eq. 43 becomes

$$
\begin{aligned}
& c_{\tau}^{(0)}(q)= \\
& -\frac{\pi n}{4} \sum_{\tau^{\prime}} F_{\tau, \tau^{\prime}}(q) \sum_{\tau^{\prime \prime}} M_{\tau^{\prime}} M_{\tau^{\prime \prime}} l_{\tau^{\prime}} l_{\tau^{\prime \prime}}\left(d_{\tau^{\prime}}+d_{\tau^{\prime \prime}}\right) c_{\tau^{\prime \prime}}^{(0)}(q)
\end{aligned}
$$

Rewriting in terms of dimensionless quantities, we obtain

$$
\mathbf{c}_{0}(q)=-\frac{\pi \tilde{n} M}{4(1+\tilde{M})\left(1+\tilde{M} \tilde{l}^{2} \tilde{d}\right)} \mathbf{F}(q) \mathbf{G}_{0} \mathbf{c}_{0}(q)
$$

with

$$
\begin{aligned}
\mathbf{G}_{0}=\left[\begin{array}{cc}
1 & \frac{1}{2} \tilde{M} \tilde{l}(1+\tilde{d}) \\
\frac{1}{2} \tilde{M} \tilde{l}(1+\tilde{d}) & \tilde{M}^{2} \tilde{l}^{2} \tilde{d}
\end{array}\right] \\
\text { and } \quad \mathbf{c}_{0}(q)=\left(\begin{array}{c}
c_{\mathrm{A}}^{(0)} \\
c_{\mathrm{B}}^{(0)}
\end{array}\right)(q) .
\end{aligned}
$$

The elements of $\mathbf{F}(q)$ are

$$
\begin{aligned}
& F_{\mathrm{A}, \mathrm{A}}=\frac{1}{M_{\mathrm{A}}^{2}}\left(M_{\mathrm{A}}+\right. \\
& \left.\frac{2}{1-j_{0}\left(q l_{\mathrm{A}}\right)}\left\{\left(M_{\mathrm{A}}-1\right)-\frac{j_{0}\left(q l_{\mathrm{A}}\right)-\left(j_{0}\left(q l_{\mathrm{A}}\right)\right)^{M_{\mathrm{A}}}}{1-j_{0}\left(q l_{\mathrm{A}}\right)}\right\}\right), \\
& F_{\mathrm{A}, \mathrm{B}}=F_{\mathrm{B}, \mathrm{A}}=\left(\frac{1}{M_{\mathrm{A}}} \frac{1-\left(j_{0}\left(q l_{\mathrm{A}}\right)\right)^{M_{\mathrm{A}}}}{1-j_{0}\left(q l_{\mathrm{A}}\right)}\right) \times \\
& \left(\frac{1}{M_{\mathrm{B}}} \frac{1-\left(j_{0}\left(q l_{\mathrm{B}}\right)\right)^{M_{\mathrm{B}}}}{1-j_{0}\left(q l_{\mathrm{B}}\right)}\right)
\end{aligned}
$$

and

$$
\begin{aligned}
& F_{\mathrm{B}, \mathrm{B}}=\frac{1}{M_{\mathrm{B}}^{2}}\left(M_{\mathrm{B}}+\right. \\
& \left.\frac{2}{1-j_{0}\left(q l_{\mathrm{B}}\right)}\left\{\left(M_{\mathrm{B}}-1\right)-\frac{j_{0}\left(q l_{\mathrm{B}}\right)-\left(j_{0}\left(q l_{\mathrm{B}}\right)\right)^{M_{\mathrm{B}}}}{1-j_{0}\left(q l_{\mathrm{B}}\right)}\right\}\right) .
\end{aligned}
$$

Again there are two solutions for this $2 \times 2$ eigenvalue problem but this time the plus sign (see again Eq. 34) yields the physical bifurcation density, $\tilde{n}_{\mathrm{mps}}$, for the microseparated phase (mps),

$$
\begin{aligned}
& \tilde{n}_{\mathrm{mps}}=-\frac{4(1+\tilde{M})\left(1+\tilde{M} \tilde{l}^{2} \tilde{d}\right)}{\pi M} \times \\
& \left(\operatorname{tr}\left(\mathbf{F}(q) \mathbf{G}_{0}\right)+\sqrt{\operatorname{tr}^{2}\left(\mathbf{F}(q) \mathbf{G}_{0}\right)-4 \operatorname{det} \mathbf{F}(q) \operatorname{det} \mathbf{G}_{0}}\right) \\
& /\left(2 \operatorname{det} \mathbf{F}(q) \operatorname{det} \mathbf{G}_{0}\right) .
\end{aligned}
$$

Apart from the approximations made in formulating the model, Sec. [1] Eqs. 40 and 41] constitute the only two further approximations. From Eq. 49 it is observed directly that the spinodal density of the microseparated phase scales with $1 / M$, contrary to the nematic spinodal, Eq. 35] which does not depend on $M$ in this representation. Consequently, for long enough polymers the system will always become unstable with respect to the microseparated phase. Furthermore, we note that for infinitely long chains $(M \rightarrow \infty)$ the approximations become exact (and the density needs to be rescaled, $\tilde{n} M$ ). If the chains are not long, the approximations, Eqs. 40 and 41 will not be valid. An interesting case are e.g. rod-coil copolymers where $M_{\mathrm{A}}=1$ and $M_{\mathrm{B}}$ is large. The type-A rods will tend to form a smectic which the type- $\mathrm{B}$ tails are likely to stabilize [50, 51]. In this case, Eq. [39] has to be solved numerically or in some other (approximate) way. Moreover, the ordering of the type-A rods is 
then likely to be dominated by an orientationally ordered density fluctuation, e.g. possibly $\exp [i \mathbf{q} \cdot \mathbf{r}] P_{2}(\hat{q} \cdot \hat{\omega})$, instead of the simple exp $[i \mathbf{q} \cdot \mathbf{r}]$ which we have in the present case. Finally, we note that the specification of the geometry is contained in the matrix $\mathbf{F}(q)$. Using other geometries, e.g. ABABAB... repeating multiblock copolymers or branched geometries, do not change Eqs. 39 or 49 but only the form of $\mathbf{F}(q)$ (the only requirement is that there are no closed loops within the polymers [32]).

\section{THE GAUSSIAN LIMIT}

In this section we will construct a consistent limit for infinitely long chains of our model. There are several reasons for this approach. First of all, there is a large body of literature dealing with so-called Gaussian chains, i.e. polymers which are coarse-grained on the level of the radius of gyration, and we want to make contact with those treatments [1, 33]. Secondly, we do not fully control the quality of the approximations, Eqs. 40 and 41 made for chains of finite length. It is clear, however, that these approximations become exact for infinitely long polymers. Finally, by introducing this limiting case the number of effective model parameters is reduced, resulting in a conceptually simpler system. The limit of $M_{\mathrm{A}}, M_{\mathrm{B}} \rightarrow \infty$ does require that some of the other parameters be rescaled as well. Additionally, we want to take this limit in such a way that the nematic and microseparated bifurcation densities remain of the same order of magnitude so that we can compare them. This extra requirement is non-trivial as can be seen from Eqs. 35 and 49 because $\tilde{n}_{\text {mps }}$ scales with $1 / M$ and thus vanishes for long polymers. We can cure this divergence in a somewhat unconventional way by letting the difference in thickness of the A and B segments vanish, $\tilde{d} \rightarrow 1$. In this way, the incentive for MPS is much reduced and $\tilde{n}_{\text {mps }}$ "pulled up" to nonzero densities comparable to $\tilde{n}_{\text {nem }}$. Summarizing, we take the limits

$$
M_{\mathrm{A}} \rightarrow \infty, l_{\mathrm{A}} \rightarrow 0 \text { and } \tilde{d} \rightarrow 1
$$

whilst $M_{\mathrm{A}} l_{\mathrm{A}}^{2}$ and $M_{\mathrm{A}}(1-\tilde{d})^{2}$ remain finite. Furthermore, we keep the ratios $\tilde{M}$ and $\tilde{l}$ fixed, such that the type-B segments are subject to the same limit. Next, in order for the Onsager approximation to still be valid, $d_{\mathrm{A}}$ needs to remain much smaller than $l_{\mathrm{A}}$ and therefore needs to go to zero even faster. This is corrected by letting the number density of chains go to infinity in order to keep total strength of the interaction, i.e. the total excluded volume constant. So additionally we have

$$
d_{\mathrm{A}} \rightarrow 0 \text { and } n \rightarrow \infty
$$

with $2 n M_{\mathrm{A}} l_{\mathrm{A}}^{2} d_{\mathrm{A}}$ and therefore also $\tilde{n}=2 n\left(M_{\mathrm{A}} l_{\mathrm{A}}^{2} d_{\mathrm{A}}+\right.$ $\left.M_{\mathrm{B}} l_{\mathrm{B}}^{2} d_{\mathrm{B}}\right)$ finite.

In the Gaussian limit, the relevant length scale is the radius of gyration or equivalently, the mean-square endto-end distance. The mean-square end-to-end distance is defined as

$$
x^{2}=\sum_{k, k^{\prime}}<l_{k} \hat{\omega}_{k} \cdot l_{k^{\prime}} \hat{\omega}_{k^{\prime}}>
$$

where $<>$ denotes the average over a single chain 55 . In a freely-jointed chain there is no orientational correlation between the segments so for our block copolymers, the mean-square end-to-end distance is simply $x^{2}=M_{\mathrm{A}} l_{\mathrm{A}}^{2}+M_{\mathrm{B}} l_{\mathrm{B}}^{2}$. This allows us to define the dimensionless wavenumber as $\tilde{q}=q x$.

Our reduced model has three parameters, $\tilde{M}, \tilde{l}$ governing the composition and the relative size of the copolymeric blocks and $\tilde{\Delta} \equiv M_{A}(1-\tilde{d})^{2}$ describing the remaining difference in thickness between the two components and hence effectively setting the incentive for demixing.

In the Gaussian limit, the determinant of $\mathbf{G}_{2}$ goes to zero, $\operatorname{det} \mathbf{G}_{2}=-\frac{1}{4} \tilde{M} \tilde{l}^{2}(1-\tilde{d})^{2} \rightarrow 0$. Consequently, we can expand Eq 35 for small $\operatorname{det} \mathbf{G}_{2}$ and we obtain for the nematic bifurcation density in the Gaussian limit,

$$
n_{\mathrm{nem}}=\frac{32\left(1+\tilde{M} \tilde{l}^{2}\right)}{\pi \operatorname{tr} \mathbf{G}_{2}(\tilde{d}=1)}=\frac{32}{\pi},
$$

which, conveniently, is a constant independent on the model parameters. Setting the first element of the eigenvector to one, $\mathbf{c}_{\text {nem }}=\left(1, c_{\text {nem }}\right)$, this is very simple in the Gaussian limit, $c_{\text {nem }}=\tilde{l}$. Therefore, at the nematic bifurcation the $\mathrm{B}$ segments are $\tilde{l}$ times more strongly orientationally ordered than the A segments.

Concerning MPS, we first calculate the elements of $\mathbf{F}$ in the Gaussian limit,

$$
\begin{gathered}
F_{\mathrm{A}, \mathrm{A}}=\frac{12}{q_{\mathrm{A}}^{2}}\left\{1-\frac{6}{q_{\mathrm{A}}^{2}}\left(1-\mathrm{e}^{-q_{\mathrm{A}}^{2} / 6}\right)\right\} \\
F_{\mathrm{A}, \mathrm{B}}=F_{\mathrm{B}, \mathrm{A}}=\frac{6}{q_{\mathrm{A}}^{2}}\left(1-\mathrm{e}^{-q_{\mathrm{A}}^{2} / 6}\right) \frac{6}{q_{\mathrm{B}}^{2}}\left(1-\mathrm{e}^{-q_{\mathrm{B}}^{2} / 6}\right) \\
F_{\mathrm{B}, \mathrm{B}}=\frac{12}{q_{\mathrm{B}}^{2}}\left\{1-\frac{6}{q_{\mathrm{B}}^{2}}\left(1-\mathrm{e}^{-q_{\mathrm{B}}^{2} / 6}\right)\right\}
\end{gathered}
$$

with

$$
q_{\mathrm{A}}^{2}=\frac{\tilde{q}^{2}}{1+\tilde{M} \tilde{l}^{2}} \text { and } q_{\mathrm{B}}^{2}=\frac{\tilde{q}^{2} \tilde{M} \tilde{l}^{2}}{1+\tilde{M} \tilde{l}^{2}}
$$

The determinant of $\mathbf{G}_{0}$ also goes to zero, $\operatorname{det} \mathbf{G}_{0}=$ $-\frac{1}{4} \tilde{M}^{2} \tilde{l}^{2}(1-\tilde{d})^{2} \rightarrow 0$. Next, expanding Eq. 49 for small $\operatorname{det} \mathbf{G}_{0}$ as well, we obtain for the bifurcation density of MPS in the Gaussian limit,

$$
\begin{array}{r}
\tilde{n}_{\mathrm{mps}}=-\lim _{\tilde{d} \rightarrow 1} \frac{4(1+\tilde{M})\left(1+\tilde{M} \tilde{l}^{2} \tilde{d}\right)}{\pi M} \frac{\operatorname{tr}\left(\mathbf{F} \mathbf{G}_{0}\right)}{\operatorname{det} \mathbf{F} \operatorname{det} \mathbf{G}_{0}} \\
=\frac{16(1+\tilde{M})\left(1+\tilde{M} \tilde{l}^{2}\right)}{\pi \tilde{\Delta}^{2} \tilde{M}^{2} \tilde{l}^{2}} \frac{\operatorname{tr}\left(\mathbf{F} \tilde{\mathbf{G}}_{0}\right)}{\operatorname{det} \mathbf{F}},
\end{array}
$$




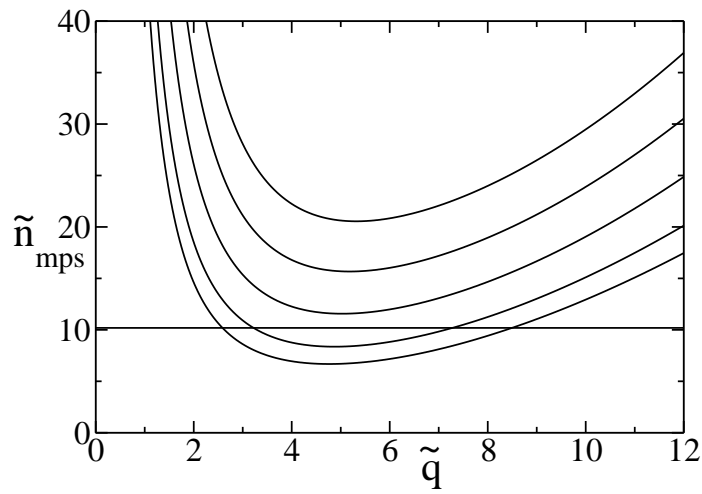

FIG. 2: Bifurcation density for the microseparated phase vs. the magnitude of the wave vector for $\tilde{l}=1$ and $\tilde{\Delta}=4$ and $\tilde{M}=\{5,4,3,2,1\}$ (from top to bottom). The nematic bifurcation density $\tilde{n}_{\text {nem }}=32 / \pi \approx 10$ and has no wave dependence but is drawn as a straight line for comparison. Due to symmetry $(\{\tilde{M}, \tilde{l}\} \rightarrow\{1 / \tilde{M}, 1 / \tilde{l}\})$ the curves of $\tilde{M}$ are the same for $1 / \tilde{M}$.

with $\tilde{\mathbf{G}}_{0}=\lim _{\tilde{d} \rightarrow 1} \mathbf{G}_{0}$,

$$
\tilde{\mathbf{G}}_{0}=\left[\begin{array}{cc}
1 & \tilde{M} \tilde{l} \\
\tilde{M} \tilde{l} & \tilde{M}^{2} \tilde{l}^{2}
\end{array}\right] .
$$

Additionally, we note the symmetry in the $\mathrm{A}$ and $\mathrm{B}$ types, i.e. the following transformation $\{\tilde{\Delta}, \tilde{M}, \tilde{l}\} \rightarrow$ $\{\tilde{\Delta}, 1 / \tilde{M}, 1 / \tilde{l}\}$ leaves the results unchanged. Again, writing the eigenvector as follows $\mathbf{c}_{\mathrm{mps}}=\left(1, c_{\mathrm{mps}}\right)$ we obtain a simple expression in the Gaussian limit $c_{\mathrm{mps}}=-1 / \tilde{M} \tilde{l}$. This is the relative order of component $\mathrm{B}$ over $\mathrm{A}$ at bifurcation. The minus sign is due to the difference of $\pi$ in phase between the density waves of $\mathrm{A}$ and $\mathrm{B}$, i.e. where the density of $\mathrm{A}$ is enhanced the density of $\mathrm{B}$ is depressed $\left(\mathrm{e}^{i \pi}=-1\right)$. The absolute value $1 / \tilde{M} \tilde{l}$ is ratio of amplitudes of the two waves. The matrix F contains the correlations within the polymer and is seen to feature the so-called Debije functions, $g_{D}(x)=$ $(2 / x)(1-(1 / x)(1-\exp [-x]))$ reflecting the Gaussian character of the correlations. In the Leibler approach 33] these appear in a similar way and therefore, the correlations are treated on the same level.

\section{RESULTS}

\section{A. Bifurcation Density}

In Fig. 2 we have plotted the analytical bifurcation density of the microseparated phase, Eq. (56) as a function of the wave vector for various values of $\tilde{M}$. Most importantly, all curves have a minimum for a certain wave vector. Interpreting the bifurcation point as the spinodal, where the isotropic fluid phase changes from being stable to unstable, the system becomes first unstable for fluctuations with a wave length corresponding to the minimum

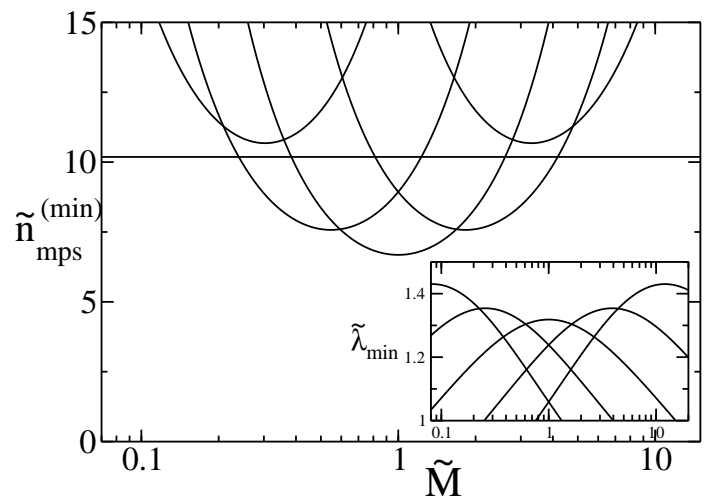

FIG. 3: The minimum bifurcation density for the microseparated phase vs. $\log \tilde{M}$ for $\tilde{\Delta}=4$ and $\tilde{l}=\{0.25,0.5,1,2,4\}$ (right to left). The nematic bifurcation density $\tilde{n}_{\text {nem }}=$ $32 / \pi \approx 10$ is constant and drawn as a straight line for comparison. Inset: the wave length for which the bifurcation density of the microseparated phase is a minimum, $\tilde{\lambda}_{\min }=2 \pi / \tilde{q}_{\min }$ vs. $\tilde{M}$ for the same parameters; $\tilde{\Delta}=4$ and $\tilde{l}=\{0.25,0.5,1,2,4\}$ (right to left).

density. We have also plotted the nematic bifurcation density, being a constant independent of the wavenumber $\tilde{q}$, in Fig. 2 For the curves which lie totally above the horizontal line, the system becomes unstable with respect to the nematic phase at the density $\tilde{n}=\tilde{n}_{\text {nem }}=32 / \pi$. For a curve of which the minimum reaches below the horizontal line, the system becomes unstable with respect to a microseparated phase with wave length $\tilde{\lambda}_{\text {min }}=2 \pi / \tilde{q}_{\text {min }}$ at the minimum density $\tilde{n}=\tilde{n}_{\mathrm{mps}}^{(\mathrm{min})}$. In Fig. 2 we have set the $\mathrm{A}$ and $\mathrm{B}$ segments to equal length, $\tilde{l}=1$ and the demixing parameter is $\tilde{\Delta}=4$. Starting with an asymmetric polymer, $\tilde{M}=5$, MPS only occurs for high densities. Making the polymer more symmetric and decreasing $\tilde{M}$ to one, the curves shift to lower densities until at $\tilde{M}=1$ it is at its lowest position. Upon a further decrease $\tilde{M}$ following the sequence $\tilde{M}=\left\{1, \frac{1}{2}, \frac{1}{3}, \frac{1}{4}, \frac{1}{5}\right\}$, we again follow the same curves in Fig. 22 due to the symme$\operatorname{try}\{\tilde{\Delta}, \tilde{M}, \tilde{l}\} \rightarrow\{\tilde{\Delta}, 1 / \tilde{M}, 1 / \tilde{l}\}$ and the choice $\tilde{l}=1$, but now from the bottom to the top.

We have numerically determined the minimum of the MPS bifurcation density with respect to the wave vector, Eq. (56) and plotted that in Fig. 3 as a function of $\tilde{M}$ for a few different $\tilde{l}$. We observe the same trend we saw in Fig. 2] for very asymmetric polymers, $\tilde{M} \ll 1$, the minimum MPS bifurcation density is very high. Increasing $\tilde{M}$, the bifurcation density goes down until a certain value $\tilde{M}$ (depending on $\tilde{l}$ ) after which it goes up again. As shown in Fig. 3 some of the curves reach below the horizontal line marking the stability limit of the isotropic phase towards nematic ordering. Consequently, in the intermediate region the microseparated phase is probably the most stable phase, whereas for the more asymmetric polymers MPS is likely to be preempted by the nematic phase. Furthermore, there is also a dependence on $\tilde{l}$, i.e. increasing the asymmetry between the A and B segments, 
the curves shift to higher densities. Again, we note that the two curves for $\tilde{l}=0.5$ and $\tilde{l}=2$ can be mapped onto each other due to symmetry in the model parameters. In the inset of Fig. [3 we have plotted the value of the wave length $\tilde{\lambda}=2 \pi / \tilde{q}$ corresponding to $\tilde{n}_{\mathrm{mps}}^{(\min )}$ vs. $\tilde{M}$. There is a rough correspondence as a function of $\tilde{M}$ in that the lower the MPS bifurcation densities in Fig. B connect to the higher wave lengths in Fig. B(inset). In general, we have observed that the wave length for which the MPS is the stable phase (over the nematic) roughly lie between 1 and 1.5 times the mean end-to-end distance $x$, i.e. the polymers get somewhat stretched at the phase transition.

\section{B. Phase Diagrams}

Figs. 4 and 5 present the phase diagrams. We have numerically computed the model parameters for which the minimum MPS bifurcation density equals the nematic bifurcation density. In Fig. 固 the phase diagram is given in terms of $\tilde{M}$ vs. $\tilde{\Delta}$ for equal length segments, $\tilde{l}=1$. For low $\tilde{\Delta}$ the incentive for MPS is too weak and the MPS bifurcation densities are higher than the nematic ones everywhere. Increasing $\tilde{\Delta}$, the MPS becomes stable for $\tilde{M}=1$ (totally symmetric diblock copolymer) and increasing $\tilde{\Delta}$ further the range of $\tilde{M}$ for stable MPS grows correspondingly. This is not surprising as the MPS bifurcation density scales simply with $1 / \tilde{\Delta}$. The inset of Fig. 4 shows the vertical scale logarithmically to show the symmetry with respect to $\tilde{M} \rightarrow 1 / \tilde{M}$. In Fig. [5] the phase diagram is plotted for $\tilde{M}$ vs. $\tilde{l}$. The same observation as in Sec. VIA can be made: for asymmetric polymers, the nematic phase is the most stable whereas for more symmetric ones the MPS can be stable. Of course the amount of area in Fig. [5] depends sensitively on $\tilde{\Delta}$. Note that $\tilde{l}$ plays a very similar role as $\tilde{M}$. Naively, one might expect that a difference in lengths of the segments would also increase the tendency to microphase separate or at least not counteract to it. However, this is not the case, and only the difference in thickness, even though only infinitesimally small in the Gaussian limit, drives the occurence of MPS, in line with earlier work on binary mixtures of rods 20. Potentially, length differences between the component rods could drive MPS within the nematic phase, but probing this would require the numerical solutions to the full self-consistency problem, currently beyond our scope.

\section{The Density Shift along the Polymer}

The elements of the eigenvectors at the bifurcation as discussed in Secs. IVB and IVC contain information about the relative amplitude of the nascent ordering with respect to the the homogeneous and isotropic parent phase. However, by construction these quantities were averaged over all segments either of type A or B. In

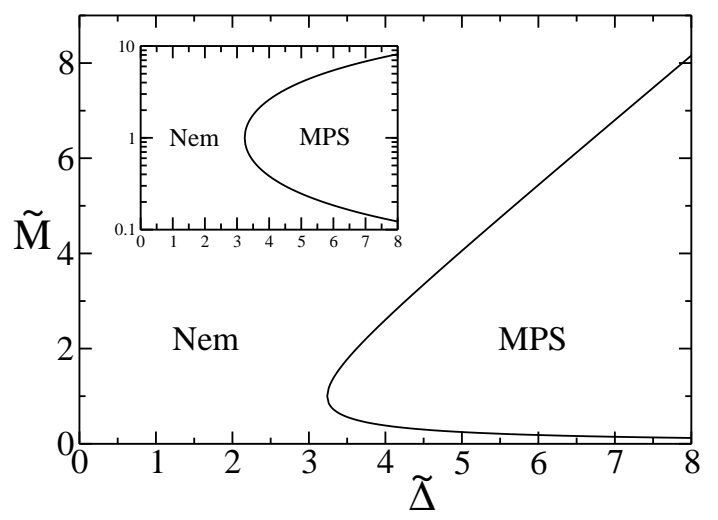

FIG. 4: Phase diagram, $\tilde{M}$ vs. $\tilde{\Delta}$ for $\tilde{l}=1$. For the region marked with "Nem", the lowest bifurcation density is the nematic and for the region marked with "MPS" this is the microseparated phase. The inset is the same phase diagram except that the vertical axis is logarithmic to show the symmetry with respect to $\{\tilde{M}, \tilde{l}\} \rightarrow\{1 / \tilde{M}, 1 / \tilde{l}\}$.

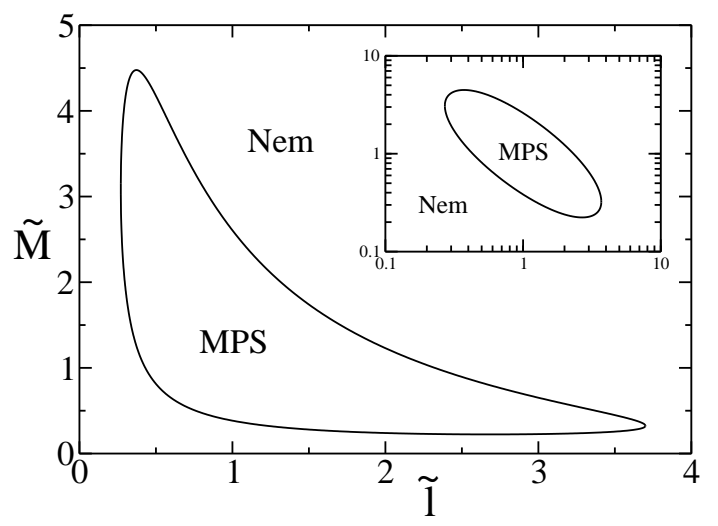

FIG. 5: Phase diagram, $\tilde{M}$ vs. $\tilde{l}$ for $\tilde{\Delta}=4$. For the region marked with "Nem", the lowest bifurcation density is the nematic and for the region marked with "MPS" this is the microseparated phase. The inset is the same phase diagram except that the axes are logarithmic to show the symmetry with respect to $\{\tilde{M}, \tilde{l}\} \rightarrow\{1 / \tilde{M}, 1 / \tilde{l}\}$.

case of the nematic ordering, this also coincides exactly with the order of each of the segments individually as there is no orientational coupling between the segments and these therefore behave as being independent. However, in case of MPS, there clearly is a spatial coupling between the segments and, consequently, one would expect a different degree of ordering e.g. for segments which are close to the free end and those which are close to the joint. Those close the joint are be subjected to two counteracting density waves and will order less than those at the free ends. In order to to quantify these effects we have to compute the components of the $M$-dimensional vector $c_{m}^{(0)}$ (Eq. (43)). In appendix [C] we explain how these are obtained from the type-averaged 2-dimensional eigenvectors by means of an additional quantity: the half type-averaged matrix $\mathbf{F}^{\prime}$. In the Gaussian limit, this $M$ - 


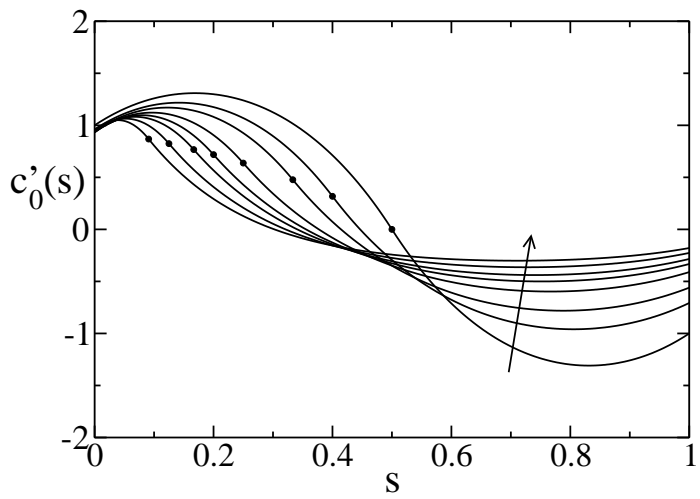

FIG. 6: Relative order along the polymer at bifurcation in the microseparated phase, $c_{\mathrm{A}}^{(0)}(s)$ for $s \in[0,1 /(1+\tilde{M})]$ and $c_{\mathrm{B}}^{\prime(0)}(s)$ for $s \in[1 /(1+\tilde{M}), 1]$. Parameters are $\tilde{\Delta}=4, \tilde{l}=1$ and $\tilde{M}=\{1,1.5,2,3,4,5,7,10\}$ (increasing in the direction of the arrow). The normalization is such that the averages over $c_{\mathrm{A}}^{\prime(0)}(s \in \mathrm{A})$ and $c_{\mathrm{B}}^{\prime(0)}(s \in \mathrm{B})$ equal $c_{\mathrm{A}}^{(0)}=1$ and $c_{\mathrm{B}}^{(0)}=$ $-1 /(\tilde{M} \tilde{l})$ respectively. The full circles indicate the "joints" of the A and B parts at $s=1 /(1+\tilde{M})$.

dimensional vector reduces to the following 2-dimensional eigenvector (with a prime),

$$
\mathbf{c}_{0}^{\prime}(s)=\left(\begin{array}{c}
c_{\mathrm{A}}^{(0)}(s \in A) \\
c_{\mathrm{B}}^{\prime(0)}(s \in B)
\end{array}\right) .
$$

which now depends, on the continuous label $s \in[0,1]$, where $s \in\left[0, \frac{1}{1+M}\right]$ implies $s \in \mathrm{A}$ and $s \in\left[\frac{1}{1+M}, 1\right]$ implies $s \in$ B. In Figs. 6 and 7 we plot the components of $\mathbf{c}_{0}^{\prime}(s)$ along the polymer (as a function of $s$ ) for increasing $\tilde{M}$ and $\tilde{l}$ respectively. The demixing parameter is taken to be $\tilde{\Delta}=4$.

In Fig. [6] we start from the symmetric case, $\tilde{M}=1$ and $\tilde{l}=1$ where the profile is also symmetric around $s=0.5$. All A segments have positive order and all B segments have negative order and the average of $\mathrm{A}$ and $\mathrm{B}$ is +1 and -1 respectively as expected. Increasing $\tilde{M}$, the $\mathrm{B}$ part of the polymer becomes larger than the A part and the joint shifts to the left. The normalization remains such that average order of the A segments is still 1 and that of the B segments is $-1 / \tilde{M}$. However, it is remarkable that the B segments close to the joint obtain a positive order with increasing $\tilde{M}$, i.e. they order with respect to the density wave of A instead of that of B. This is due to the fact that in the polymer there is much more material from the B part. Consequently, this effect becomes stronger for larger $\tilde{M}$. In Fig. [] we start again from the symmetric case, $\tilde{M}=1$ and $\tilde{l}=1$. Subsequently, the ratio of lengths $\tilde{l}$ is increased and we see that the derivative of the profile to $s$ jumps at the joint. Furthermore, also here, the joint shifts to positive values and the A segments have a much more constant profile than the B segments. By increasing $\tilde{l}$ while $\tilde{M}$ remains constant one effectively increases the amount of material in the B part of the polymer. Therefore, it is not surprising that the

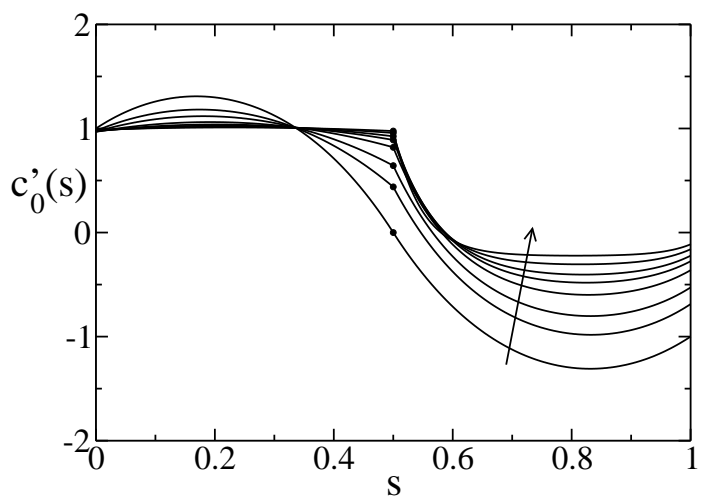

FIG. 7: Relative order along the polymer at bifurcation in the microseparated phase, $c_{\mathrm{A}}^{(0)}(s)$ for $s \in[0,1 /(1+\tilde{M})]$ and $c_{\mathrm{B}}^{\prime(0)}(s)$ for $s \in[1 /(1+\tilde{M}), 1]$. Parameters are $\tilde{\Delta}=4, \tilde{M}=$ 1 and $\tilde{l}=\{1,1.5,2,3,4,5,7,10\}$ (increasing in the direction of the arrow). The normalization is such that the averages over $c_{\mathrm{A}}^{\prime(0)}(s \in \mathrm{A})$ and $c_{\mathrm{B}}^{\prime(0)}(s \in \mathrm{B})$ equal $c_{\mathrm{A}}^{(0)}=1$ and $c_{\mathrm{B}}^{(0)}=$ $-1 /(\tilde{M} \tilde{l})$ respectively. The full circles indicate the "joints" of the A and B parts at $s=1 /(1+\tilde{M})$.

point of zero order shifts to the right. Additionally, the B segments are much longer and therefore the spatial correlations persist over larger $s$ explaining the more smooth profile on the B side. It has to be noted that some of the profiles (especially for higher values of $\tilde{M}$ and $\tilde{l}$ in Figs. [6] and 7) are taken at bifurcation densities far above the nematic bifurcation. We have nevertheless included them, being instructive in explaining the observed trends.

\section{CONCLUSION}

We have considered a fluid of freely-jointed hard diblock copolymers. The two polymer blocks A and B consist of slender Onsager rods of different dimensions interacting via hard body repulsion only. We apply a DFT approach in the second virial approximation from first principles, and analytically construct local solutions to the stationarity equations, by means of a stability (bifurcation) analysis of the isotropic phase. Spatial as well as orientational degrees of freedom are taken into account and consequently we obtain the spinodal densities for both the microseparated and the nematic phases. It is shown that for long polymers the system always becomes unstable with respect to the microseparated phase first. Consequently, this means that entropy can induce MPS in much the same way as it has been found to induce other forms of spontaneous ordering before. Furthermore, the mechanism is determined solely by the (difference in) dimensions of the rods and therefore has a conceptually simple geometric origin.

In order to make contact with the literature on thermotropic block copolymers we take the limit of infinitely long polymers in which the approximations become exact. In addition, by assuming a vanishing difference in 
thickness of the two types of rods, we can still study the competition of the microseparated with the nematic phase. We present phase diagrams in terms of model parameters showing the regions of stable microseparated or nematic ordering. We also present the order along the polymer at the bifurcation of the microseparated phase.

In the present study, we have solved the stationarity equations up to first-order in a bifurcation analysis. This yields, apart from the location of the spinodal or bifurcation density, only the magnitude of the density wave vector and the spherical harmonic mode to which the isotropic solution becomes unstable. However, the symmetry of the bifurcating microseparated solution is typically determined by one or more mutually independent (but equally long) vectors spanning the periodic phase (e.g. lamellar, hexagonal or bcc). In order to obtain information on the mutual orientation of these lattice vectors, and thus on the symmetry of the phase, a higher order bifurcation analysis should be performed [31, 56, 57]. From these higher order bifurcation equations, it is also possible to determine whether the phase transition is of first or second order and in the latter case one could in principle go on to construct the full equilibrium solution far away from the bifurcation point [31].

We have not checked the validity of the approximations, Eqs. 40 and 41 for finite values of $M$. However, we can make a crude estimate, a posteriori, by concluding from Fig. 3 that the bifurcating wave length is of the order of the mean square end-to-end distance, $\tilde{\lambda}=\lambda / x \sim 1$. Consequently, the wave vector is approximately, $\tilde{q}=2 \pi / \tilde{\lambda} \sim 2 \pi$ and if we assume for a moment that the type- $\mathrm{A}$ rods and type- $\mathrm{B}$ rods are more or less equally long, then the mean-square end-to-end distance is $x^{2} \sim M l_{\mathrm{A}}^{2}$. This in turn implies that the next order corrections in Eqs. 40 and 41 will be of order $\left(\frac{1}{2} q l_{\mathrm{A}}\right)^{2} \sim\left(\frac{1}{2} \times 2 \pi\right)^{2} / M \sim 10 / M$. (In fact the first order correction in $\left(\frac{1}{2} q l_{\mathrm{A}}\right)$ in Eq. 41 does not contribute to the value of the bifurcation density, but only to the form of the eigenfunction.) Consequently, already for this crude test case, the length of the polymer should be at least longer than $10(M>10)$ in order for the corrections to be smaller than the leading term. This suggests that much higher values of $M$ are required for the present approach to yield quantitative agreement with the "true" behaviour.

In any case, it would be very interesting to extend the present approach to finite values of $M$. However, this is not straightforward, as the correlations within the chain would become non-Gaussian. One strategy could be to solve Eq. 39] directly numerically but this could become tedious for large numbers of segments. Another strategy would be to make an expansion in $1 / M$ using the Gaussian limit as a reference state. This last route was followed by Fredrickson and Helfand [35] for Leibler's diblock copolymers and the results were confirmed by simulations 37]. Indeed, there is a need for such a betterthan-Gaussian treatment, especially when the typical ordering length scales are of the same sizes as the components, e.g. for side chain liquid crystalline polymers forming a smectic [58, 59].

As already mentioned in the introduction, there is as yet no experimental system exhibiting MPS due to the mechanism described in this paper. However, considering the ongoing progress in the field of bioengineering [26, 60], it may become possible to prepare such a system. We mention again the possibility of long and thin polymers connected to TMV rods in an appropriate solvent. The solvent may be a problem as we have the double requirement that the polymers are at their $\theta$ point and that at the same time the TMV rods still act as hard particles. Still, such a system of entropic rod-coil copolymers could be directly compared to the simulation studies of Refs. 50, 51]. Additionally, it would be described by Eq. 39] which would than have to be solved for the case of $M_{\mathrm{A}}=1$ and $M_{\mathrm{B}}$ large. In a more general context,it becomes increasingly clear that entropyinduced effects play a prominent role in vivo [61], and it may be that similar mechanisms as described here prevent demixing tendencies due to local constraints 60]. On the other hand, the mechanism may also be of relevance in thermotropic systems where the two components of block copolymers also have short-range anisotropic repulsions which are usually of different range. In any case, observing entropy-induced microphase separation in monodisperse systems would certainly be an interesting experimental challenge.

\section{Acknowledgments}

The authors would like to thank M. Schmidt and D. Lukatsky for critically reading the manuscript. This work is part of the research program of the "Stichting voor Fundamenteel Onderzoek der Materie" (FOM), which is financially supported by the "Nederlandse Organisatie voor Wetenschappelijk Onderzoek" (NWO). PPFW would like to thank the Heinriche-HeineUniversität Düsseldorf for hospitality, where part of writing this paper was finished.
[1] F. S. Bates and G. H. Fredrickson. Annu. Rev. Phys. Chem., 21:525, 1990.

[2] F. S. Bates. Science, 251:898, 1991.

[3] M. W. Matsen and F. S. Bates. Macromolecules, 29:1091, 1996.
[4] V. Shibaev and L. Lam, editors. Liquid Crystalline and Mesomorphic Polymers. Springer, 1994.

[5] D. Demus, J. Goodby, G. W. Gray, H.-W. Spiess, and V. Vill, editors. Handbook of Liquid Crystals: Vol. 3. High Molecular Weight Liquid Crystals. Wiley-VCH, 
1998.

[6] W. M. Gelbart, D. Roux, and A. Ben-Shaul. Micelles, Membranes, Microemulsions and Monolayers. Springer, Berlin, 1994.

[7] G. Gompper and M. Schick. Self-Assembling Amphiphilic Systems. Academic Press, London, 1994.

[8] M. Adams, Z. Dogic, S. L. Keller, and S. Fraden. Nature (London), 393:349, 1998.

[9] M. Adams and S. Fraden. Biophys. J., 74:669, 1998.

[10] T. Koda, M. Numajiri, and S. Ikeda. J. Phys. Soc. Jpn., 65:3551, 1996.

[11] Z. Dogic, D. Frenkel, and S. Fraden. Phys. Rev. E, 62:3925, 2000.

[12] B. J. Alder and T. E. Wainwright. J. Chem. Phys., 27:1208, 1957.

[13] L. Onsager. Ann. N. Y. Acad. Sci., 51:627, 1949.

[14] S. Asakura and F. Oosawa. J. Chem. Phys., 22:1255, 1954.

[15] S. Asakura and F. Oosawa. J. Polym. Sci., 33:183, 1958.

[16] A. Vrij. Pure and Appl. Chem., 48:471, 1976.

[17] W. C. K. Poon. J. Phys.: Cond. Matt., 14:R859, 2002.

[18] H. N. W. Lekkerkerker and A. Stroobants. Nature (London), 393:305, 1998.

[19] R. P. Sear and G. Jackson. J. Chem. Phys., 103:8684, 1995.

[20] R. van Roij and B. Mulder. Phys. Rev. E, 54:6430, 1996.

[21] R. P. Sear and B. M. Mulder. J. Chem. Phys., 105:1, 1996.

[22] H. Bosetti and A. Perera. Phys. Rev. E, 63:021206, 2001.

[23] M. Dijkstra and R. van Roij. Phys. Rev. E, 56:5594, 1997.

[24] R. van Roij, B. Mulder, and M. Dijkstra. Physica A, 261:374, 1998.

[25] S. Fraden. Phase transitions in colloidal suspensions of virus particles. In M. Baus, L. F. Rull, and J.-P. Ryckaert, editors, Observation, Prediction and Simulation of Phase Transitions in Complex Fluids, page 113. Kluwer Academic Publishers, 1994.

[26] Z. Dogic and S. Fraden. Phil. Trans. R. Soc. Lond. A, 359:997, 2001.

[27] A. R. Khokhlov and A. N. Semenov. Physica A, 108:546, 1981.

[28] A. R. Khokhlov and A. N. Semenov. Physica A, 112:605, 1982.

[29] G. J. Vroege and H. N. W. Lekkerkerker. Rep. Prog. Phys., 55:1241, 1992.

[30] P. P. F. Wessels and B. M. Mulder. Soft Materials, 1:313, 2003.

[31] R. F. Kayser Jr. and H. J. Raveché. Phys. Rev. A, 17:2067, 1978.

[32] P. P. F. Wessels and B. M. Mulder. to be submitted to Physica A.

[33] L. Leibler. Macromolecules, 13:1602, 1980.

[34] A. N. Semenov. Sov. Phys. JETP, 61:733, 1985.

[35] G. H. Fredrickson and E. Helfand. J. Chem. Phys., 87:697, 1987.

[36] M. W. Matsen and M. Schick. Phys. Rev. Lett., 72:2660, 1994.

[37] H. Fried and K. Binder. J. Chem. Phys., 94:8349, 1991.

[38] U. Micka and K. Binder. Macromol. Theory Simul., 4:419, 1995.

[39] A. N. Semenov and S. V. Vasilenko. Sov. Phys. JETP, 63:70, 1986.

[40] R. Hołyst and M. Schick. J. Chem. Phys., 96:730, 1992.

[41] D. R. M. Williams and A. Halperin. Phys. Rev. Lett.,
71:1557, 1993.

[42] C. Singh, M. Goulian, A. J. Liu, and G. H. Fredrickson. Macromolecules, 27:2974, 1994.

[43] R. A. Sones and R. G. Petschek. Phys. Rev. E, 50:2906, 1994.

[44] R. R. Netz and M. Schick. Phys. Rev. Lett., 77:302, 1996.

[45] M. Reenders and G. ten Brinke. Macromolecules, 35:3266, 2002.

[46] R. Hołyst. Phys. Rev. A, 42:3438, 1990.

[47] P. G. Bolhuis and D. Frenkel. Physica A, 244:45, 1997.

[48] M. Schmidt and C. von Ferber. Phys. Rev. E, 64:051115, 2001.

[49] D. Düchs and D. E. Sullivan. J. Phys.: Cond. Matt., 14:12189, 2002.

[50] J. S. van Duijneveldt and M. P. Allen. Mol. Phys., 92:855, 1997.

[51] A. Casey and P. Harrowell. J. Chem. Phys., 110:12183, 199.

[52] R. Evans. Chapter 3, density functionals in the theory of nonuniform fluids. In D. Henderson, editor, Fundamentals of Inhomogeneous Fluids, page 85. Dekker, New York, 1992.

[53] M. P. Allen, G. T. Evans, D. Frenkel, and B. M. Mulder. Adv. Chem. Phys., 86:1, 1993.

[54] J. P. Hansen and I. R. McDonald. Theory of Simple Liquids. Academic Press, London, 2nd edition, 1986.

[55] P.-G. de Gennes. Scaling Concepts in Polymer Physics. Cornell University Press, Ithaca and London, 1st edition, 1979.

[56] B. M. Mulder. Phys. Rev. A, 35:3095, 1987.

[57] B. M. Mulder. Phys. Rev. A, 39:360, 1989.

[58] A. Donald and A. Windle, editors. Liquid Crystalline Polymers. Cambridge University Press, 1992.

[59] W. Renz and M. Warner. Phys. Rev. Lett., 56:1268, 1986.

[60] T. Surrey, F. Nédélec, S. Leibler, and E. Karsenti. Science, 292:1167, 2001.

[61] T. Z. Rizvi. J. Mol. Liq., 106:43, 2003.

\section{APPENDIX A: THE FOURIER TRANSFORMED SEGMENT-SEGMENT MAYER FUNCTION}

The Mayer function $\phi_{k, k^{\prime}}$ of two cylindrical rodlike segments $k$ (with dimensions $l_{k}, d_{k}$ and coordinates $\left(\mathbf{r}_{k}, \hat{\omega}_{k}\right)$ ) and $k^{\prime}$ (with $l_{k^{\prime}}, d_{k^{\prime}}$ and $\left(\mathbf{r}_{k^{\prime}}, \hat{\omega}_{k^{\prime}}\right)$ ) interacting via a hardcore potential (i.e. $=\infty, 0$ if overlap/no overlap) is given by

$$
\phi_{k, k^{\prime}}\left(\mathbf{r}_{k}-\mathbf{r}_{k^{\prime}}, \hat{\omega}_{k}, \hat{\omega}_{k^{\prime}}\right)= \begin{cases}-1 & \text { if overlap } \\ 0 & \text { if no overlap }\end{cases}
$$

We decompose the spatial vector $\mathbf{r}_{k, k^{\prime}}=\mathbf{r}_{k}-\mathbf{r}_{k^{\prime}}$ in terms of the orientations,

$$
\mathbf{r}_{k, k^{\prime}}=x_{k} \hat{\omega}_{k}+x_{k^{\prime}} \hat{\omega}_{k^{\prime}}+x_{k, k^{\prime}} \hat{\omega}_{k, k^{\prime}}
$$

with $\hat{\omega}_{k, k^{\prime}}=\left(\hat{\omega}_{k} \times \hat{\omega}_{k^{\prime}}\right) /\left|\hat{\omega}_{k} \times \hat{\omega}_{k^{\prime}}\right|$ the unit vector in the perpendicular direction. There is overlap between the two rods for the following ranges of the coefficients, $x_{k} \in$ $\left[-l_{k} / 2, l_{k} / 2\right], x_{k^{\prime}} \in\left[-l_{k^{\prime}} / 2, l_{k^{\prime}} / 2\right]$ and $x_{k, k^{\prime}} \in\left[-\left(d_{k}+\right.\right.$ $\left.\left.d_{k^{\prime}}\right) / 2,\left(d_{k}+d_{k^{\prime}}\right) / 2\right]$. Next, the Fourier transform of the 
Mayer function $\hat{\phi}_{k, k^{\prime}}$ is given by

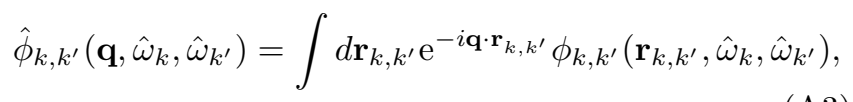

where the volume of the infinitesimal element is given by $d \mathbf{r}_{k, k^{\prime}}=\left|\hat{\omega}_{k} \times \hat{\omega}_{k^{\prime}}\right| d x_{k} d x_{k^{\prime}} d x_{k, k^{\prime}}$. Consequently,

$$
\begin{aligned}
& \hat{\phi}_{k, k^{\prime}}\left(\mathbf{q}, \hat{\omega}_{k}, \hat{\omega}_{k^{\prime}}\right)= \\
& -\left|\hat{\omega}_{k} \times \hat{\omega}_{k^{\prime}}\right| \int_{-l_{k} / 2}^{l_{k} / 2} d x_{k} \int_{-l_{k^{\prime}} / 2}^{l_{k^{\prime}} / 2} d x_{k^{\prime}} \int_{-\left(d_{k}+d_{k^{\prime}}\right) / 2}^{\left(d_{k}+d_{k^{\prime}}\right) / 2} d x_{k, k^{\prime}} \times \\
& \exp \left[-i\left(x_{k} \mathbf{q} \cdot \hat{\omega}_{k}+x_{k^{\prime}} \mathbf{q} \cdot \hat{\omega}_{k^{\prime}}+x_{k, k^{\prime}} \mathbf{q} \cdot \hat{\omega}_{k, k^{\prime}}\right)\right] \\
& =-l_{k} l_{k^{\prime}}\left(d_{k}+d_{k^{\prime}}\right)\left|\hat{\omega}_{k} \times \hat{\omega}_{k^{\prime}}^{\prime}\right| j_{0}\left(\frac{1}{2} l_{k} \mathbf{q} \cdot \hat{\omega}_{k}\right) \times \\
& \quad j_{0}\left(\frac{1}{2} l_{k^{\prime}} \mathbf{q} \cdot \hat{\omega}_{k^{\prime}}^{\prime}\right) j_{0}\left(\frac{1}{2}\left(d_{k}+d_{k^{\prime}}\right) \mathbf{q} \cdot \hat{\omega}_{k, k^{\prime}}\right) \quad(\mathrm{A} 4)
\end{aligned}
$$

with the spherical Bessel function of zeroth order given by $j_{0}(x)=\sin x / x$. In the Onsager limit of very slender rods, $l_{k}, l_{k^{\prime}} \gg d_{k}, d_{k^{\prime}}$ while $l_{k} l_{k^{\prime}}\left(d_{k}+d_{k^{\prime}}\right)$ stays finite. In our system, we expect the wave length of the microseparated phase to be at least of the order of the lengths of the segments (although for large number of segments it is even much larger). Consequently, in this case, $\left|\left(d_{k}+d_{k^{\prime}}\right) \mathbf{q}\right| \ll 1$ and we use the leading order, which is $j_{0}\left(\frac{1}{2}\left(d_{k}+d_{k^{\prime}}\right) \mathbf{q} \cdot \hat{\omega}_{k, k^{\prime}}\right)=1$. Then, our final result for the Mayer function is

$$
\begin{aligned}
\hat{\phi}_{k, k^{\prime}}\left(\mathbf{q}, \hat{\omega}_{k}, \hat{\omega}_{k^{\prime}}\right)= & -l_{k} l_{k^{\prime}}\left(d_{k}+d_{k^{\prime}}\right)\left|\hat{\omega}_{k} \times \hat{\omega}_{k^{\prime}}^{\prime}\right| \times \\
& j_{0}\left(\frac{1}{2} l_{k} \mathbf{q} \cdot \hat{\omega}_{k}\right) j_{0}\left(\frac{1}{2} l_{k^{\prime}} \mathbf{q} \cdot \hat{\omega}_{k^{\prime}}^{\prime}\right) .
\end{aligned}
$$

\section{APPENDIX B: THE EIGENFUNCTIONS OF $\hat{\phi}_{k, k^{\prime}}\left(\hat{\omega} \cdot \hat{\omega}^{\prime}\right)$ FOR $\mathbf{q}=0$}

For $\mathbf{q}=0$, the Fourier transformed Mayer function is

$$
\begin{aligned}
\hat{\phi}_{k, k^{\prime}}\left(\hat{\omega} \cdot \hat{\omega}^{\prime}\right) & =-l_{k} l_{k^{\prime}}\left(d_{k}+d_{k^{\prime}}\right)\left|\hat{\omega} \times \hat{\omega}^{\prime}\right| \\
& =-l_{k} l_{k^{\prime}}\left(d_{k}+d_{k^{\prime}}\right) \sqrt{1-\left(\hat{\omega} \cdot \hat{\omega}^{\prime}\right)^{2}}
\end{aligned}
$$

and is therefore uniaxial, i.e. dependent on a single planar angle $\gamma=\arccos \left(\hat{\omega} \cdot \hat{\omega}^{\prime}\right)$. Therefore, we can expand it in terms of Legendre polynomials

$$
\hat{\phi}_{k, k^{\prime}}\left(\hat{\omega} \cdot \hat{\omega}^{\prime}\right)=-l_{k} l_{k^{\prime}}\left(d_{k}+d_{k^{\prime}}\right) \sum_{j=0}^{\infty} \frac{2 j+1}{4 \pi} s_{j} P_{j}\left(\hat{\omega} \cdot \hat{\omega}^{\prime}\right),
$$

with $s_{j}=2 \pi \int_{-1}^{1} d x \sqrt{1-x^{2}} P_{j}(x)$. Then, using the decomposition in terms of spherical harmonics $Y_{j, i}$, we can rewrite this as

$$
\begin{aligned}
& \hat{\phi}_{k, k^{\prime}}\left(\hat{\omega} \cdot \hat{\omega}^{\prime}\right)=-l_{k} l_{k^{\prime}}\left(d_{k}+d_{k^{\prime}}\right) \times \\
& \sum_{j=0}^{\infty} \sum_{i=-j}^{j} \frac{2 j+1}{4 \pi} s_{j} Y_{j, i}(\hat{\omega} \cdot \hat{z}) Y_{j, i}^{*}\left(\hat{\omega}^{\prime} \cdot \hat{z}\right),
\end{aligned}
$$

with the asterisk denoting the complex conjugate and $\hat{z}$ some unit vector. It is now directly seen that the Legendre polynomials are eigenfunctions of $\hat{\phi}_{k, k^{\prime}}\left(\hat{\omega} \cdot \hat{\omega}^{\prime}\right)$

$$
\int d \hat{\omega}^{\prime} \hat{\phi}_{k, k^{\prime}}\left(\hat{\omega} \cdot \hat{\omega}^{\prime}\right) P_{j}\left(\hat{\omega}^{\prime} \cdot \hat{z}\right)=-l_{k} l_{k^{\prime}}\left(d_{k}+d_{k^{\prime}}\right) s_{j} P_{j}(\hat{\omega} \cdot \hat{z}) .
$$

\section{APPENDIX C: SPATIAL ORDER WITHIN THE POLYMER}

It is possible to calculate the bifurcating order within the polymer. In case of freely-jointed chains in the nematic phase this is trivial as this exactly $c_{\tau}^{(2)}$ for a segment of type $\tau$. However, in case of MPS, segments of type A close to the "joint" with B segments will typically be more affected by the B part of the polymer than segments of type A far away from the joint. This order within the polymer can be obtained by calculating the elements of the $M$-dimensional vector $\mathbf{c}_{0}^{\prime}$ with elements $c_{m}^{(0)}$ and $m \in\{1, \cdots, M\}$ (see Eq. 43). Therefore we proceed by defining the matrix $\mathbf{F}^{\prime}$ (with a prime)

$$
F_{m \in \tau, \tau^{\prime}}^{\prime}=\frac{1}{M_{\tau^{\prime}}} \sum_{k^{\prime} \in \tau^{\prime}} F_{m, k^{\prime}}
$$

where the average is only performed over the second label and therefore $F_{m, \tau^{\prime}}^{\prime}$ is $M \times 2$ dimensional. Then, if the bifurcation density for the microseparated phase $\tilde{n}_{\mathrm{mps}}$ and the corresponding eigenvector $\mathbf{c}_{\mathrm{mps}}$ has been calculated beforehand (from Eq. 49), $\mathbf{c}_{0}^{\prime}$ can be computed by evaluating

$$
\mathbf{c}_{0}^{\prime}=-\frac{\pi \tilde{n}_{\mathrm{mps}} M}{4(1+\tilde{M})\left(1+\tilde{M} \tilde{l}^{2} \tilde{d}\right)} \mathbf{F}^{\prime} \mathbf{G}_{0} \mathbf{c}_{\mathrm{mps}} .
$$

The elements of $\mathbf{F}^{\prime}$ are given by

$$
\begin{aligned}
& F_{m \in \mathrm{A}, \mathrm{A}}^{\prime}= \\
& \frac{1}{M_{\mathrm{A}}}\left(1+\frac{2-\left(j_{0}\left(q l_{\mathrm{A}}\right)\right)^{m-1}-\left(j_{0}\left(q l_{\mathrm{A}}\right)\right)^{M_{\mathrm{A}}-m}}{1-j_{0}\left(q l_{\mathrm{A}}\right)}\right) \\
& F_{m \in \mathrm{A}, \mathrm{B}}^{\prime}=\frac{1}{M_{\mathrm{B}}}\left(j_{0}\left(q l_{\mathrm{A}}\right)\right)^{M_{\mathrm{A}}-m} \frac{1-\left(j_{0}\left(q l_{\mathrm{B}}\right)\right)^{M_{\mathrm{B}}}}{1-j_{0}\left(q l_{\mathrm{B}}\right)} \\
& F_{m \in \mathrm{B}, \mathrm{A}}^{\prime}=\frac{1}{M_{\mathrm{A}}}\left(j_{0}\left(q l_{\mathrm{B}}\right)\right)^{m-M_{\mathrm{A}}-1} \frac{1-\left(j_{0}\left(q l_{\mathrm{A}}\right)\right)^{M_{\mathrm{A}}}}{1-j_{0}\left(q l_{\mathrm{A}}\right)} \\
& F_{m \in \mathrm{B}, \mathrm{B}}^{\prime}= \\
& \frac{1}{M_{\mathrm{B}}}\left(1+\frac{2-\left(j_{0}\left(q l_{\mathrm{B}}\right)\right)^{m-1-M_{\mathrm{A}}}-\left(j_{0}\left(q l_{\mathrm{B}}\right)\right)^{M-m}}{1-j_{0}\left(q l_{\mathrm{B}}\right)}\right)
\end{aligned}
$$


where $m \in\left\{1, \cdots, M_{\mathrm{A}}\right\}$ when $m \in A$ and $m \in\left\{M_{\mathrm{A}}+\right.$ $1, \cdots, M\}$ when $m \in B$. For each of these elements again holds that the average of $m$ yields the matrix $\mathbf{F}$ (see above Eq. 45] and Eq. 48), i.e.

$$
F_{\tau, \tau^{\prime}}=\frac{1}{M_{\tau}} \sum_{m \in \tau} F_{m \in \tau, \tau^{\prime}}^{\prime}
$$

In the Gaussian limit, we have to define a continuous "label", $s=m / M$, with $m$ and $M$ going to infinity such that $s$ keeps its value. Consequently, $s \in[0,1]$ and $\mathbf{F}^{\prime}$ becomes

$$
\begin{aligned}
& F_{\mathrm{A}, \mathrm{A}}^{\prime}(s \in \mathrm{A})=\frac{6}{q_{\mathrm{A}}^{2}}\left(2-\exp \left[-\frac{q_{\mathrm{A}}^{2}}{6} s(1+\tilde{M})\right]\right.\left.\exp \left[-\frac{q_{\mathrm{A}}^{2}}{6}(1-s(1+\tilde{M}))\right]\right) \\
& F_{\mathrm{A}, \mathrm{B}}^{\prime}(s \in \mathrm{A})=\frac{6}{q_{\mathrm{B}}^{2}}\left(1-\exp \left[-\frac{q_{\mathrm{B}}^{2}}{6}\right]\right) \times \\
& \quad \exp \left[-\frac{q_{\mathrm{A}}^{2}}{6}(1-s(1+\tilde{M}))\right] \\
& F_{\mathrm{B}, \mathrm{A}}^{\prime}(s \in \mathrm{B})=\frac{6}{q_{\mathrm{A}}^{2}}\left(1-\exp \left[-\frac{q_{\mathrm{A}}^{2}}{6}\right]\right) \times \\
& \exp \left[-\frac{q_{\mathrm{B}}^{2}}{6}\left(s \frac{1+\tilde{M}}{\tilde{M}}-\frac{1}{\tilde{M}}\right)\right]
\end{aligned}
$$

$$
\begin{aligned}
F_{\mathrm{B}, \mathrm{B}}^{\prime}(s \in \mathrm{B})=\frac{6}{q_{\mathrm{B}}^{2}} & \left(2-\exp \left[-\frac{q_{\mathrm{B}}^{2}}{6}\left(s \frac{1+\tilde{M}}{\tilde{M}}-\frac{1}{\tilde{M}}\right)\right]\right. \\
& \left.-\exp \left[-\frac{q_{\mathrm{B}}^{2}}{6}(1-s) \frac{1+\tilde{M}}{\tilde{M}}\right]\right) \quad(\mathrm{C} 11)
\end{aligned}
$$

where $s \in\left[0, \frac{1}{1+M}\right]$ when $s \in \mathrm{A}$ and $s \in\left[\frac{1}{1+M}, 1\right]$ when $s \in \mathrm{B}$. Note that in the Gaussian limit $\mathbf{F}^{\prime}$ is simply a $2 \times 2$ matrix, however, with $s$-dependence. Consequently, unlike $\mathbf{F}, \mathbf{F}^{\prime}$ is not symmetric. And additionally, also the $M$-dimensional eigenvector becomes 2-dimensional,

$$
\mathbf{c}_{0}^{\prime}(s)=\left(\begin{array}{c}
c_{\mathrm{A}}^{\prime(0)}(s \in A) \\
c_{\mathrm{B}}^{\prime(0)}(s \in B)
\end{array}\right) .
$$

Finally, it has to be noted that in the Gaussian limit, first the product of $\mathbf{G}_{0}$ and $\mathbf{c}_{\mathrm{mps}}$ has to be taken and only then the limit can be applied to $\left(\mathbf{G}_{0} \mathbf{c}_{\mathrm{mps}}\right)$. 